\title{
Supersaturation Variability and Cirrus Ice Crystal Size Distributions
}

\author{
B. KÄRCHER, A. DÖRNBRACK, AND I. SÖLCH \\ Institut für Physik der Atmosphäre, DLR Oberpfaffenhofen, Wessling, Germany
}

(Manuscript received 30 December 2013, in final form 31 March 2014)

\begin{abstract}
Small-scale dynamical variability affects atmospheric supersaturation and therefore the development of ice clouds via uptake of water vapor on ice crystals. This variability and its implications for ice growth are difficult to capture experimentally and theoretically. By interpreting supersaturation as a stochastic variable, the authors examine the average temporal behavior of, and the link between, supersaturation fluctuations and ice crystal size distributions in upper-tropospheric cirrus clouds. The authors classify cirrus types according to their ability to dampen supersaturation fluctuations owing to depositional growth of cloud ice and study how size distributions in them respond to supersaturation variability, investigating the possibility of the occurrence of ice-supersaturated states within cirrus. Typical time scales for growth and damping impacts on supersaturation are minutes and minutes to hours, respectively, and are highly variable among cirrus types and within single clouds. Transient deviations from saturated equilibrium states can occur depending on the ice crystal number concentration and size and on the strength of the small-scale dynamical forcing. Supersaturation preferentially occurs in cloud regions with few small ice crystals. The authors demonstrate that supersaturation fluctuations in very thin tropical tropopause cirrus create long-lived supersaturated states. Furthermore, they potentially generate few large ice crystals, broadening size distributions, and significantly enhance water mass fluxes due to sedimentation. Although not studied here, they may also allow new ice crystals to nucleate. Implications of these findings for those clouds to dehydrate air entering the lower stratosphere are discussed and future research needs are outlined.
\end{abstract}

\section{Introduction}

Tropospheric ice clouds exist in different temperature regimes: the cold cloud regime, below about $235 \mathrm{~K}$, where liquid water in supercooled cloud or aerosol droplets freezes spontaneously, and the mixed-phase cloud regime at higher temperatures extending up to the melting point, where water droplets and ice crystals might coexist. As pure ice clouds, cirrus reside in the upper troposphere. Mixed-phase clouds are thermodynamically instable and turn, at lower altitudes, into pure ice clouds in the presence of suitable ice nuclei. Here, we focus on the cold cloud (cirrus) regime.

A nuanced understanding of the physical properties of cirrus is vital to obtain more robust predictions of ice cloud radiative forcing (Stephens et al. 1990). Radiative transfer models and remote sensing retrieval algorithms need to employ realistic ice particle size distributions

Corresponding author address: Bernd Kärcher, Deutsches Zentrum für Luft- und Raumfahrt, Institut für Physik der Atmosphäre, Oberpfaffenhofen, 82234 Wessling, Germany.

E-mail: bernd.kaercher@dlr.de
(PSDs) in order to infer instantaneous cloud radiative flux changes. Models with coarse resolution achieved considerable complexity in representing microphysical processes in large-scale clouds (Collins and Satoh 2009; Lohmann and Schwartz 2009), but the need remains to parameterize PSDs in order to evaluate optical properties and microphysical process rates. Among all factors controlling ice crystal growth by uptake of water vapor, ice supersaturation, ${ }^{1}$ a measure of the amount of water vapor above or below the ice-saturated (thermodynamic equilibrium) value, is of central importance.

Climate predictions rely on a comprehensive understanding of a suite of dynamical, radiative, and microphysical processes determining the moisture content at the entry to the tropical stratosphere (Solomon et al.

\footnotetext{
${ }^{1}$ We use "supersaturation" $s$ to denote conditions in which ice crystals either grow $(s>0)$ or sublimate $(s<0)$. The term "growth," as employed here, includes cases in which ice crystals sublimate unless stated otherwise. When expressed as a percentage, the ice saturation ratio $(s+1)$ is identical to the relative humidity over ice.
} 
2010). Uptake of water vapor on ice crystals in very cold cirrus, forming frequently in the tropical tropopause layer below $195 \mathrm{~K}$, critically affects the amount of water vapor in the middle atmosphere (Randel and Jensen 2013). Air entering the stratosphere may at times be ice supersaturated. At the same time, the PSD determines the number of ice crystals with terminal fall velocities large enough to settle out of the cloud layer before crossing the tropopause, thereby reducing the amount of total water during ascent. Observations of frequent ice supersaturation inside those clouds provoked speculation as to the cause of such departures from equilibrium states (Peter et al. 2006). Variability in temperature (and therefore in supersaturation) unresolved in global climate models has been shown to affect the lifetimes of thin tropical tropopause cirrus (Jensen et al. 2011).

\section{a. Depositional growth of ice crystals}

Cirrus clouds are composed of ice crystals with sizes in the range between about $1 \mu \mathrm{m}$ and $1 \mathrm{~mm}$. As illustrated below, the lower part of this size range is mainly affected by depositional growth, whereby the mass and size of ice crystals increase by the deposition of water $\left(\mathrm{H}_{2} \mathrm{O}\right)$ molecules on the crystal surfaces. The $\mathrm{H}_{2} \mathrm{O}$ molecules diffuse through air toward the cloud particles on whose surfaces they are deposited.

Depositional growth of ice crystals becomes ineffective at large sizes of a few hundred micrometers, beyond which growth can proceed more efficiently via aggregation (i.e., the collision and subsequent sticking of ice crystals). Aggregation preferentially occurs in vertically thick cirrus at relatively warm temperatures, where the chances of collisions and sticking probabilities are high. Contrary to aggregation, depositional ice growth is driven directly by supersaturation of $\mathrm{H}_{2} \mathrm{O}$. It is the only process by which cirrus clouds increase their water content and equilibrate with the gas phase. Large regions of the cloud-free upper troposphere are frequently ice supersaturated (Kahn et al. 2009; Lamquin et al. 2012), particularly in the tropics.

The depositional vapor growth rate follows from classical diffusion theory (Pruppacher and Klett 1997):

$$
\frac{d r}{d t}=\frac{\nu D(p, T)}{r} \frac{e(T)}{k_{B} T} s,
$$

with time $t$, the volume of an $\mathrm{H}_{2} \mathrm{O}$ molecule in ice $\nu$, their diffusion coefficient in air $D$, air temperature $T$, air pressure $p$, the saturation vapor pressure of water vapor over ice $e$, Boltzmann's constant $k_{B}$, and the ambient ice supersaturation $s$. (See appendix $\mathrm{C}$ for a table of all symbols and acronyms, along with their definitions and units, used throughout this paper.) Equation (1) assumes ice crystals to be spherical with radii $r$ of volumeequivalent spheres and predicts that, for given $s$ and $T$, smaller particles change their size faster than larger particles, leading to a continual narrowing of the size distribution over time during growth $(s>0)$. On the other hand, sublimation $(s<0)$ creates broad, negatively skewed distributions (i.e., with tails extending toward small particle sizes).

Depending on details of how $\mathrm{H}_{2} \mathrm{O}$ molecules are incorporated into the crystal lattice, Eq. (1) is valid for $r>$ $1-10 \mu \mathrm{m}$ up to sizes for which ventilation corrections become significant (i.e., for large, rapidly falling ice crystals). At small growth efficiency, details of the deposition process (surface kinetic effects: adsorption, molecular, and thermal accommodation including the Kelvin barrier) matter. Extending Eq. (1) to properly account for such effects on realistic ice crystal surfaces remains elusive (Wood et al. 2001). On the other hand, ice crystal sizes in our study are small enough so that habit effects on the growth rate are limited; they might be included in Eq. (1) by means of a capacitance factor.

\section{b. Small-scale fluctuations of ice supersaturation}

Synoptic regimes conducive to cirrus formation vary widely, defining the large-scale environment in which ice cloud particles nucleate and develop. Cirrus clouds exhibit significant internal meso- and microscale structure (Sassen et al. 1989), including vertical air motion variability (Yang et al. 2012). Nonetheless, little is known about the characteristics of small-scale supersaturation variability in cirrus clouds and its impact on cirrus microphysical properties. Fluctuations of supersaturation inside cirrus arise from variability in $T$ and in the $\mathrm{H}_{2} \mathrm{O}$ partial pressure. Small-scale fluctuations in temperature are brought about by gravity waves and turbulence leading to rapid buoyancy oscillations. Inhomogeneities in $\mathrm{H}_{2} \mathrm{O}$ concentrations are caused by localized ice formation events, flow instabilities, and convective overturning or by sedimentation and sublimation of ice crystals.

In view of the difficulty to ascribe cause to and properly characterize supersaturation variability, we have recently proposed a probabilistic model describing supersaturation variability in air parcels containing ice crystals (Kärcher 2012). The basic idea is that supersaturation is a stochastic variable that evolves because of a random forcing and that the fluctuations are damped owing to uptake of $\mathrm{H}_{2} \mathrm{O}$ molecules on growing ice crystals, reducing the $\mathrm{H}_{2} \mathrm{O}$ partial pressure toward ice saturation. The air parcels are assumed to experience random perturbations of water vapor and temperature in a way that supersaturation fluctuations are driven into a time-evolving Gaussian distribution. The variance of 
the probability density function (PDF) of supersaturation fluctuations grows rapidly with time approaching a stationary value that is determined by the damping rate. As examined in more detail in the present study, depositional growth of ice crystals is an important microphysical damping mechanism for supersaturation fluctuations in cirrus. With increasing amounts of cloud ice, stationarity is approached more rapidly, and damping reduces the supersaturation fluctuations more effectively (Kärcher and Burkhardt 2008). Stationary microscopic states may include slowly varying parameters of the large-scale cloud environment.

The probabilistic part of the model is based on an exponential temporal correlation of supersaturation fluctuations (colored noise). Compared to white noise, the damped colored-noise model offers (i) a nonzero autocorrelation time of the fluctuations as one more degree of freedom, important for the description of many physical processes (Hänggi and Jung 1995), and (ii) a fluctuation variance that approaches a finite value after decorrelation owing to prescribed damping processes. The fluctuation intensity is constrained by the variance of supersaturation fluctuations in cloud-free (undamped) conditions (Kärcher 2012). In this model, $s$ is split into an ensemble ${ }^{2}$ mean $\bar{s}$ and a random part $\varphi: s=\bar{s}+\varphi$. Our expectation is that PSDs in cirrus show features-for instance, changes in the width of the distribution indicating the degree of size dispersion-that can be traced back to the fluctuating growth component.

Ensemble effects of unresolved supersaturation fluctuations on the diffusional growth of cloud water droplets ("stochastic condensation") have first been studied by Levin and Sedunov (1966) and discussed later both theoretically and experimentally (e.g., Bartlett and Jonas 1972; Manton 1979; Cooper 1989; Korolev 1995; Khvorostyanov and Curry 1999; Brenguier and Chaumat 2001; Jeffery et al. 2007). Along with a number of other mechanisms, turbulent fluctuations in supersaturation have been proposed to lead to a broadening of droplet size distributions in warm clouds, facilitating precipitation formation. While similarities exist to our approach (e.g., fluctuations are modeled by a kinetic equation with a damping rate depending on the mean droplet radius),

\footnotetext{
${ }^{2}$ The ensemble contains a sufficient number of air parcels such that mean values of cloud variables are properly defined. Those mean values characterize the large-scale environment in which the air parcels evolve. Each parcel experiences a different realization of the random supersaturation fluctuation histories along its atmospheric trajectory. Our work addresses the associated response of the size distributions of small ice crystals between air parcels with different supersaturation histories.
}

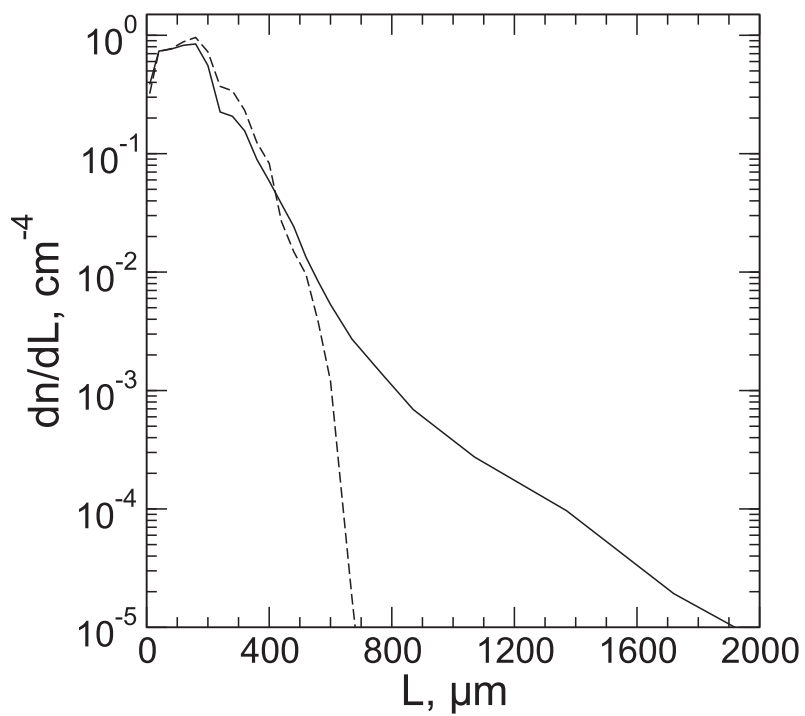

FIG. 1. Cirrus PSDs vs maximum ice crystal dimension from cloud-resolving simulations of a midlatitude cirrus cloud including depositional growth and aggregation (solid curve, $\bar{L} \simeq 150 \mu \mathrm{m}$ ) and without aggregation (dashed curve, $\bar{L} \simeq 160 \mu \mathrm{m}$ ). The PSDs are averaged over the whole volume in the mature cloud $(3 \mathrm{~h}$ after formation, the cloud depth is $4 \mathrm{~km}$ including fall streaks as shown in Fig. 3). Large ice crystals, generated by aggregation, represent about $1 \%$ of the total number concentration of ice crystals with $L>500 \mu \mathrm{m}$.

time scales for growth of particles and damping of the fluctuations are longer and supersaturations are larger in cirrus than in warm clouds. Therefore, the nonequilibrium physics of cold, pure ice clouds differs significantly from that of warm, liquid-phase clouds, so that separate studies of "stochastic deposition" in cirrus appear to be justified.

\section{c. Cirrus ice crystal size distributions}

Ice crystal size distributions vary greatly in tropospheric ice clouds (Heymsfield 2003). Ice nucleation produces micrometer-sized crystals that quickly grow to establish or maintain the small size "mode" (Ström et al. 1997), which is usually found in upper cloud regions. Large ice crystals (maximum dimension $L>100 \mu \mathrm{m}$ ) are characterized by substantial terminal fall velocities $\left(>1 \mathrm{~m} \mathrm{~s}^{-1}\right)$ and therefore predominantly populate lower cloud regions including sublimation layers (Miloshevich and Heymsfield 1997). PSDs skewed toward large particle sizes may be obtained when averaging individual PSDs over a large volume of cloud, especially in the vertical.

Figure 1 depicts such a PSD (solid curve) $d n / d L$ averaged over a deep, stratiform cirrus cloud at one particular time during its evolution as simulated with a cloud-resolving (large eddy) model that has been tightly 
constrained by meteorological and microphysical observations (Sölch and Kärcher 2010); $L$ varies with the shape of the simulated ice crystals. The solid curve is the result of a reference simulation that includes aggregation (Sölch and Kärcher 2011). This simulation explains the PSD sampled on board a research aircraft during slow spiral descent fairly well. The motivation to present this PSD here is to highlight the ice crystal size range $(L<200-400 \mu \mathrm{m})$ over which depositional growth is effective.

The dashed curve in Fig. 1 is taken from a sensitivity simulation without aggregation (i.e., ice crystal growth occurred exclusively by deposition of $\mathrm{H}_{2} \mathrm{O}$ vapor). The cloud-averaged PSD taken from the simulation without aggregation exhibits much smaller positive skewness. Comparing the solid and dashed curves in Fig. 1 reveals that small ice crystals are redistributed by aggregation to fill the size range greater than $400 \mu \mathrm{m}$. Therefore, aggregation leads to an exponential tail, which may be interpreted as a second (large) ice mode. In colder and geometrically thinner cirrus, such as those prevailing at the tropical tropopause, less water vapor is available for deposition and aggregation is much less effective, and a large mode is therefore unlikely to develop.

\section{d. Scope of the present study}

Our main goal is to unravel fundamental connections between the temporal, small-scale variability in supersaturation and microphysical properties of various types of cirrus. The interaction of supersaturation fluctuations with depositional growth of ice crystals occurs in a particle size range that is controlled by water vapor diffusion. We calculate and discuss for the first time joint PDFs of ice crystal size and supersaturation, from which PSDs as well as statistics of supersaturation fluctuations in cirrus are derived.

The joint PDFs are obtained by an approximate physical model combining a depositional growth equation with a stochastic equation describing damped fluctuations of supersaturation that drive the evolution of an ice crystal population. Our results describe the average behavior of cirrus properties and supersaturation contained in an ensemble of air parcels in terms of a probability distribution around a common mean state rather than their deterministic evolution within single air parcels.

Ice crystal growth rates driven by constant supersaturation places a restriction on the evolution of cirrus clouds (Lin et al. 1998). Variability in cloud-scale supersaturation induced by temperature fluctuations are known to affect cirrus optical depth distributions and other cloud microphysical properties (Mace et al. 2001; Kärcher and Ström 2003; Hoyle et al. 2005; Kay et al. 2006). Here, we deal with supersaturation variability in a probabilistic approach. A stochastic analysis has also been applied to radar reflectivity measurements of cirrus to investigate the large-scale control of cloud radiative properties and to explore the internal structure of the latter (Ivanova and Ackerman 2009). We fix the large-scale environment (keep ensemble-mean values fixed) and study interactions between ice supersaturation and ice crystal growth directly on the small scale (within the air parcels that constitute the ensemble) by imposing small-scale supersaturation variability. We do not attempt to study those interactions with full cirrus cloud simulations [in the spirit of Jensen et al. (1994), Khvorostyanov and Sassen (1998), and Sölch and Kärcher (2011)], although we make use of such simulations in support of our analysis. We do not include effects of nucleation, sedimentation, and spatial (interparcel) mixing, but we discuss how those processes might affect our results.

As we have emphasized in earlier studies (Kärcher and Ström 2003), small-scale vertical air motion variability has also very important implications for the nucleation of ice crystals from aerosol particles with disparate freezing efficiencies, but this aspect is not pursued in the present work.

Section 2 presents the pivotal elements of our theoretical model along with a description of the numerical solution method. Section 3 discusses joint PDFs of ice crystal size and supersaturation and examines general aspects of their evolution induced by supersaturation variability. We relate our findings to observations and large-eddy simulations of cirrus clouds. Furthermore, we study tropical tropopause cirrus in more detail (section 4). We close with a summary of our key findings and discuss implications for future work (section 5).

\section{Stochastic model and numerical approach}

\section{a. Governing equations}

The stochastic differential equations describing the depositional growth of single ice crystals coupled to supersaturation variability in the reference frame of a Lagrangian air parcel read $(\bar{s}=0)$

$$
\begin{aligned}
& \frac{d \alpha}{d t}=\gamma \varphi, \quad \gamma=\frac{2 \nu D}{r_{0}^{2}} \frac{e}{k_{B} \bar{T}}, \\
& \frac{d \varphi}{d t}=-\lambda \varphi+\epsilon, \quad \lambda=4 \pi D n r_{0} .
\end{aligned}
$$

Equation (2) is identical to Eq. (1), but expressed in terms of the ice crystal surface area, $\alpha=\left(r / r_{0}\right)^{2}$, scaled by the initial mean radius $r_{0}$ of the particle population, and the characteristic areal growth time scale $1 / \gamma$. 
Furthermore, $\lambda$ is an approximate deterministic damping rate (discussed below), or inverse supersaturation quenching time (e.g., Korolev and Mazin 2003), and $\bar{T}$ is the ensemble-mean air temperature, respectively. For colored-noise-driven supersaturation, the time-dependent random forcing of $\varphi, \epsilon(t)$, is characterized by a zero mean and exponential correlation over the time $\tau$ and a Gaussian probability distribution.

Disregarding the stochastic trigger $(\epsilon=0)$ allows us to gain first insight into the damped growth process along single particle trajectories from Eqs. (2) and (3). The corresponding solution is presented in appendix A. In the remainder of this work, we seek a numerical solution including the stochastic forcing to derive the joint PDF, $P=P(\alpha, \varphi, \theta)$, of ice crystal sizes and ice supersaturation by formulating an associated, two-dimensional FokkerPlanck equation:

$$
\frac{\partial P}{\partial \theta}=\underbrace{-\zeta \varphi \frac{\partial P}{\partial \alpha}}_{\equiv \mathcal{L}_{\alpha} P}+\underbrace{\xi \frac{\partial(\varphi P)}{\partial \varphi}+\mathcal{D} \frac{\partial^{2} P}{\partial \varphi^{2}}}_{\equiv \mathcal{L}_{\varphi} P},
$$

where we have introduced the scaled time, $\theta=t / \tau$, and the constant growth and damping factors, $\zeta=\gamma \tau$ and $\xi=$ $\lambda \tau$, respectively. The term $\mathcal{L}_{\varphi} P$ has been discussed earlier in this context (Kärcher 2012); here, we have introduced a drift term in the independent size variable $\mathcal{L}_{\alpha} P$ (Risken 1996) to complete our basic equation. The intrinsic correlation time $\tau$ depends on the nature of the stochastic forcing and must be compared to the physical time scale of the cirrus problem (minutes to hours). In the kinetic equation [Eq. (4)], $P$ describes the joint probability of finding ice crystals with a surface area $\alpha$ at the supersaturation $\varphi$ within the respective intervals $[\alpha, \alpha+\Delta \alpha]$ and $[\varphi, \varphi+\Delta \varphi]$, averaged over many air parcels (i.e., realizations of the fluctuations). The dimensionless diffusivity in $\varphi$ space $\mathcal{D}$ is time dependent for colored noise and depends also on the damping factor:

$$
\mathcal{D}(\theta)=\frac{\sigma_{\mathrm{cf}}^{2} / 2}{\xi+1}\left[1-e^{-(\xi+1) \theta}\right] .
$$

The variance of the supersaturation fluctuations in cloud-free air $\sigma_{\mathrm{cf}}^{2}$ is used to constrain $\mathcal{D}$ (Kärcher 2012) and therefore the total power contained in the fluctuations. The PDF satisfies the four boundary conditions $P(\theta, \alpha=0, \varphi)=P(\theta, \alpha \rightarrow \infty, \varphi)=P\left(\theta, \alpha, \varphi=\varphi_{\min }\right)=$ $P(\theta, \alpha, \varphi \rightarrow \infty)=0$. The lower-limit fluctuation, $\varphi_{\min }=-1$, indicates completely dry air.

We obtain the ice supersaturation PDF, $P_{\varphi}$, the ice surface area PDF, $P_{\alpha}$, and the ice crystal radius PDF, $P_{r}$, as marginal distributions of $P$ at any time:

$$
\begin{aligned}
P_{\varphi}(\varphi, \theta) & =\int_{0}^{\infty} P(\alpha, \varphi, \theta) d \alpha, \\
P_{\alpha}(\alpha, \theta) & =\int_{\varphi_{\min }}^{\infty} P(\alpha, \varphi, \theta) d \varphi, \\
P_{r}(r, \theta) & =\frac{2 r}{r_{0}^{2}} \int_{\varphi_{\min }}^{\infty} P[\alpha(r), \varphi, \theta] d \varphi .
\end{aligned}
$$

The ice crystal PSD is given by $d n / d r=n P_{r}$, where $n$ is the total ice crystal number concentration. For simplicity, in our applications, initial joint distributions are assumed to factorize in $P_{\alpha}$ and $P_{\varphi}$.

The operator $\mathcal{L}_{\alpha} P$ describes the advection of particles in $\alpha$ space and arises from the characteristic Eq. (2). Therefore, $\partial_{\theta} P=\mathcal{L}_{\alpha} P$ corresponds to the common depositional growth equation for a population of ice crystals. The operator $\mathcal{L}_{\varphi} P$ defines a one-dimensional Fokker-Planck equation for the time-correlated, normally distributed fluctuations containing an advective and a diffusive part. The salient features of individual solutions for growth and supersaturation are presented in more detail in appendix A.

Constant $\lambda$ values are required to derive Eq. (4). The use of constant $n$ and constant $r_{0}$ in the definition of $\lambda$ in Eq. (3) is an approximation. The correct damping rate to be used in a fully self-consistent microphysical simulation involving a polydisperse ice crystal population may change over time and is given by

$$
\lambda=4 \pi D \underbrace{\int P_{r} d r}_{\leq n} \underbrace{\int r P_{r} d r}_{=r_{m}} ;
$$

this means that $\lambda$ depends on the ice crystal number (first integral) and on the associated mean radius (second integral). Depositional growth (sublimation) keeps $n$ constant but causes the mean radius $r_{m}$ to increase (decrease), so we may underestimate (overestimate) the damping strength, although fluctuations tends to average out changes in $r_{m}$. Complete sublimation leading to a loss of small ice crystals reduces $n$. Nucleation increases $n$ making $r_{m}$ smaller and tends to increase $\lambda$. Gravitational settling leads to a loss of large ice crystals, reducing $n$ and $r_{m}$. Aggregation reduces $n$ and increases $r_{m}$ slightly at the same time and would not affect $\lambda$ very much. The omission of sedimentation is justified if the corresponding changes in $\lambda$ are slow on the time scales of interest here.

The damping rate derives from the diffusional growth law and ensures that gain or loss of $\mathrm{H}_{2} \mathrm{O}$ vapor correspond to changes in the fluctuations such that the total water mass is conserved in the presence of ice crystals. 
This is easily shown by transforming Eqs. (2) and (3) to describe the rates of change of the number concentration of $\mathrm{H}_{2} \mathrm{O}$ molecules, $N_{g}=\varphi e /\left(k_{B} T\right)$ and $N_{i}=$ $4 \pi n r^{3} /(3 \nu)$, in the gas phase (relative to the equilibrium concentration) and ice phase: $d N_{g} / d t=-\lambda \varphi e /\left(k_{B} T\right)$ and $d N_{i} / d t=\lambda \varphi e /\left(k_{B} T\right)$ using $\lambda=4 \pi D n r$ at constant temperature $(T=\bar{T})$ and pressure, so $N_{g}+N_{i}=$ const. In terms of the probabilistic joint PDF, conservation of total water is reflected by $\iint P d \varphi d \alpha=1$. If an ensemble-mean supersaturation was imposed $(\bar{s} \neq 0)$, total water could only be conserved when taking the large-scale environment of the air parcel ensemble into account.

\section{b. Model parameters}

The Lagrangian autocorrelation time $\tau$ for fluctuations of supersaturation is not known. In the absence of observational estimates for $\tau$, and owing to the fact that small-scale dynamical variability must be associated with short time scales (e.g., associated with buoyancy oscillations), we may tentatively identify $\tau$ with the inverse frequency of buoyancy oscillations (a Brunt-Väisälä period). Our problem is then characterized by three dimensionless parameters: $\sigma_{\mathrm{cf}}, \xi$, and $\zeta$. Two further parameters are the total number concentration of ice crystals $n$ and their initial mean radius $r_{0}$, which we associate with distinct types of cirrus clouds (section $3 b$ ).

To facilitate the identification of specific cirrus cloud types, we compute the respective growth and damping parameters, $\gamma$ and $\lambda$, respectively. In each cloud case, $\gamma$ is calculated from Eq. (2) by prescribing $r_{0}, p$, and $T$ (the two latter are used to determine $e$ and $D$ ). Furthermore, we estimate $\lambda$ values from Eq. (3) by prescribing fixed $r_{0}$, $p$, and $T$, and constant $n$. A typical cloud-free fluctuation amplitude (standard deviation of the normally distributed fluctuations), $\sigma_{\mathrm{cf}}$ has roughly been estimated to be 0.1 when caused solely by variability in temperature (Kärcher 2012). The actual fluctuation amplitudes in cirrus clouds depend on time and the damping factor $\xi$; see Eq. (B7).

\section{c. Numerical approach}

The joint $\mathrm{PDF}, P(\alpha, \varphi, \theta)$, is discretized in scaled time $\theta$ and in $\{\alpha, \varphi\}$ space with finite differences: $P_{i j}^{k}$ denotes the discrete dependent function, the upper index signifying the discretized time, $\theta^{k}=k \Delta \theta$, and lower indices signifying the equally spaced grid points, $\alpha_{i}=$ $i \Delta \alpha(i=1, \ldots, N)$ and $\varphi_{j}=j \Delta \varphi(j=1, \ldots, M)$. Grid increments are denoted by $\Delta \alpha$ and $\Delta \varphi$. On the discrete grid, the joint PDF satisfies the boundary conditions $P_{1 j}^{k}=P_{N j}^{k}=P_{i 1}^{k}=P_{i M}^{k}=0 \forall k$.

To solve Eq. (4) by finite differences, we cast it into the following form:

$$
\frac{\partial P}{\partial \theta}+\frac{\partial\left(u_{\alpha} P\right)}{\partial \alpha}+\frac{\partial\left(u_{\varphi} P\right)}{\partial \varphi}=\mathcal{D} \frac{\partial^{2} P}{\partial \varphi^{2}},
$$

where the coefficients are defined as vector components, $\mathbf{u}=\left\{u_{\alpha}, u_{\varphi}\right\}: u_{\alpha}=\zeta \varphi$ and $u_{\varphi}=-\xi \varphi$. Equation (10) is a generalized transport (advection-diffusion) partial differential equation:

$$
\frac{\partial(\rho \Psi)}{\partial \theta}+\nabla(\rho \mathbf{u} \Psi)=\rho \mathcal{R}
$$

which can be solved numerically by schemes applied for geophysical flow solvers (Prusa et al. 2008), where $\rho$ represents the mass density of air and $\Psi$ is a meteorological variable. Here, $\Psi=P$ and $\rho=1, \mathcal{R}$ represents the diffusion term, and the divergence operator acts in $\{\alpha, \varphi\}$ phase space.

The nonoscillatory forward-in-time (NFT) approximation of Eq. (10) is

$$
P_{i}^{k+1}=\mathcal{A}_{i}\left(\mathbf{u}^{k+0.5}, \tilde{P}\right)
$$

$P_{i}^{k+1}$ denotes the solution at $\left\{\theta^{k+1}, \mathbf{x}_{i}\right\}$, with $\mathbf{x}_{i}=\{\alpha, \varphi\}$ and $\tilde{P}=P^{k}+\Delta \theta R^{k} ; R_{i}^{k}$ represents the discretized onedimensional diffusion term; and $\mathcal{A}$ is the NFT transport operator that integrates the homogeneous transport equation [i.e., $\mathcal{A}$ advects $\tilde{P}$ using the fully second-order accurate flux-form scheme multidimensional positive definite advection transport algorithm (MPDATA); Smolarkiewicz and Margolin 1998; Smolarkiewicz 2006). The diffusion term is explicitly approximated to first-order accuracy [cf. section 3.5.4 in Smolarkiewicz and Margolin (1998)]. The advantage of using MPDATA is threefold. First, MPDATA preserves the sign of the joint PDF, which remains positive in the course of the integration. Second, MPDATA is conservative. Third, the MPDATA option for divergent flow has been used to account for the truncation error term proportional to the flow divergence [cf. section 3.2.3 in Smolarkiewicz and Margolin (1998)].

\section{Results and discussion}

We first discuss the joint probability density function in order to study the key features of its evolution on the one hand and to illustrate the quality of the numerical solution on the other hand (section 3a). In a second step, we identify different cirrus cloud types and relate our results to field observations and large-eddy simulations (section 3b). We then investigate the spread of ice crystal size distributions caused by supersaturation variability (section 3c). We apply the model to explore features of PSDs in tropical tropopause cirrus and to 
judge the likelihood of transient deviations from ice equilibrium within these clouds (section 4).

\section{a. Joint distributions}

We track the evolution of the joint PDF in phase space, assuming either a double Gaussian initial distribution centered at $\alpha_{0}=\varphi_{0}=1$ with a standard deviation of 0.025 in both directions or a much narrower top-hat distribution in both $\alpha$ and $\varphi$ with respective widths equal to the grid increments, $\Delta \alpha=\Delta \varphi=0.005$. The scaled time was advanced in the simulations using $\Delta \theta=0.0005$. Imposing an initial supersaturation causes the mean size of the ice crystal population to increase; hence $\lambda$ would actually change (cf. the discussion in section 2a); however, the slight error introduced by using a fixed $\lambda$ value is not important for a discussion of general features of the model solution. Figure 2 shows the evolution of the joint PDF at three different scaled times, indicating the initial $(\theta=0)$, intermediate $(\theta=1)$, and asymptotic $(\theta=10)$ stages of evolution.

In Fig. 2a, we show the baseline evolution for moderate damping factor $(\xi=0.5)$ and intermediate growth factor $(\zeta=1)$ without fluctuations $\left(\sigma_{\mathrm{cf}}=0\right)$, assuming a Gaussian supersaturation distribution at $\theta=0$. The center of the distribution moves from initially $\varphi_{0}=1$ toward $\varphi=0$ owing to the damping, and sizes increase owing to the resulting depositional growth. Furthermore, damping causes the PDF to squeeze in the $\varphi$ direction until it collapses to a thin line segment on top of the $\varphi=0$ axis. Over time, its extension in $\alpha$ direction increases substantially, because the initial PDF is spread over a (albeit small) range of $\varphi$ values. The supersaturation distribution causes some ice crystals with the same $\alpha$ but higher (lower) $\varphi$ to grow faster (slower) and the joint PDF to assume an asymmetric shape during its evolution. (This behavior is consistent with Fig. A1, which illustrates a similar case for a single-valued initial $P_{\varphi}$ distribution.) Mathematically, it follows from the advective velocity in Eq. (10) representing a sheared flow in phase space.

Damping (squeezing) is faster and growth (stretching) is weaker for stronger damping parameter, $\xi=1$, and slower growth factor, $\zeta=0.5$, as shown in Fig. $2 b$. The effect of eliminating the initial supersaturation by setting $\varphi_{0}=0$ is depicted in Fig. 2c, in which case the distribution center remains at its initial size value $\left(\alpha_{0}=1\right)$, while the shape of $P$ evolves as in the baseline case.

The addition of persistent supersaturation fluctuations $\left(\sigma_{\mathrm{cf}}=0.1\right)$ leads to an enhanced broadening of $P$ (Fig. 2d). At the intermediate time, the stochastic forcing makes a transition from a ballistic regime, in which supersaturation fluctuations induced by the colorednoise source mostly occur in the same direction, leading to a rapid broadening of the PDF, into a random walk regime, in which fluctuations in $\varphi$ change their sign very often leading to diffusive broadening of the PDF (Kärcher 2012). The asymptotic stationary distribution results from a balance between stochastic broadening and microphysical damping and is characterized by zero mean fluctuation and constant variances.

The size spreading of the PDF is greatly enhanced relative to the baseline case (Fig. 2a) for two reasons. First, owing to the fluctuations, ice crystals with a given size experience a wide range of supersaturations (stochastic mixing), whereas in the deterministic baseline case, the evolution of crystals with a given size is controlled by the initial $\varphi$ distribution. This leads to larger excursions of $\varphi$ around its actual mean value and makes the PDF at $\theta=1$ more symmetric compared to those shown in Figs. $2 \mathrm{a}-\mathrm{c}$ at the same time. Second, large portions of the PDF at $\theta=10$ extend into the region $\varphi<$ 0 , where ice crystals shrink rapidly, causing the asymptotic PDF to tilt. The tilt angle of the final joint PDF is related to the strength of damping relative to growth and expresses the tight correlation between growing ice crystals $(d r / d t>0)$ and supersaturation $(\varphi>0)$ and between sublimating ice crystals $(d r / d t<0)$ and subsaturation $(\varphi<0)$.

The results in Fig. 2e are similar to those in Fig. 2d, but were obtained using a narrow initial box distribution in both $\alpha$ and $\varphi$ variables. Despite the difference in initial conditions, the PDF evolves in a similar manner, since the noise source causes rapid stochastic mixing and therefore broadening of the initial PDF in both cases. The shapes of the PDFs at $\theta=1$ are almost identical, indicating a weak dependence of advanced stages of evolution on initial conditions. This is also true for the asymptotic PDF $(\theta=10)$, but only if the damping is not too large.

Figure $2 \mathrm{f}$ depicts supersaturation PDFs derived from Fig. 2e at selected times, underscoring the transient nature of supersaturation within cirrus clouds (in the absence of an external forcing). The final distribution inevitably centers around the saturated (thermodynamic equilibrium) value. The time a given element of cloud stays supersaturated is on the order of a few inverse damping rates; see Eq. (B9).

Modifications would arise if formation of ice crystals was included in the model. Ice nucleation would lead to transient features in the joint PDF at small sizes and large supersaturation (similar to the initial distributions shown in Fig. A1, which might therefore be interpreted as an initial nucleation event).

\section{b. Cirrus cloud types}

Observations and simulations demonstrate that distinct differences exist in microphysical properties between 


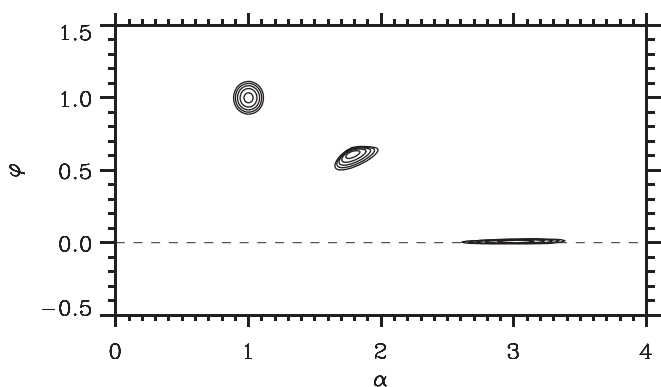

(a)

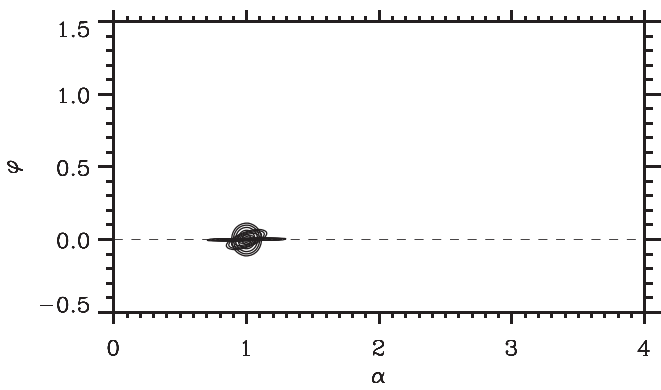

(c)

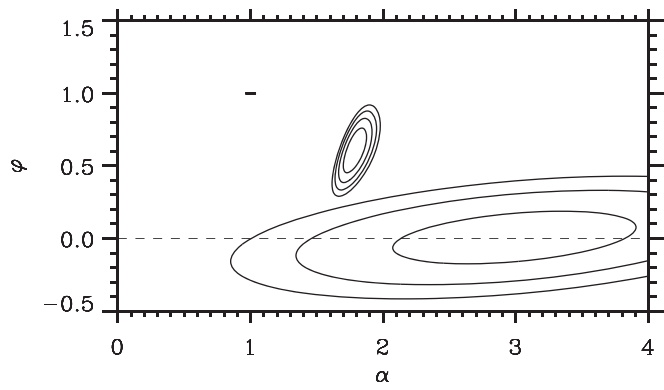

(e)

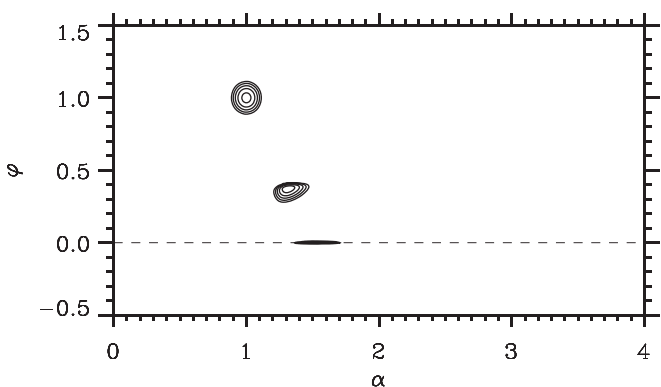

(b)

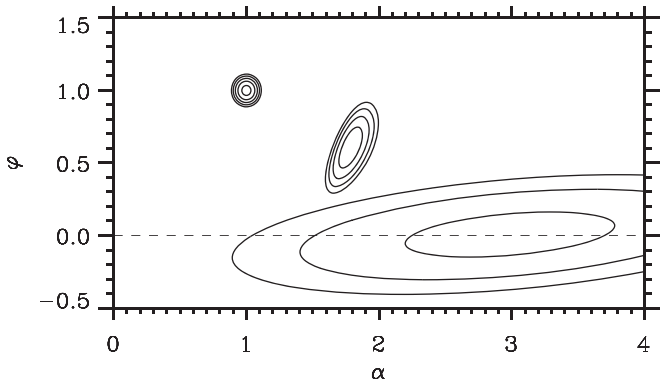

(d)

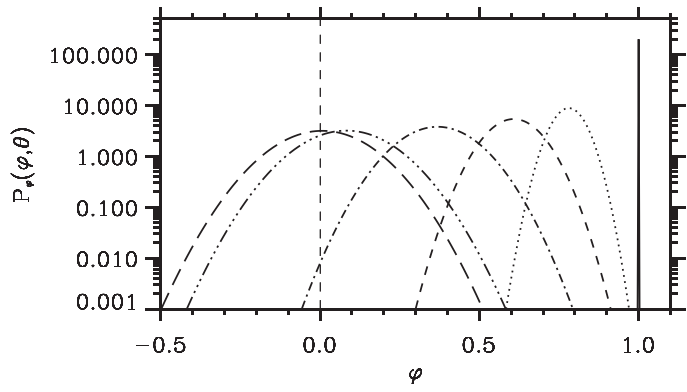

(f)

FIG. 2. Phase space evolution of joint PDFs at three different scaled times $\theta=0,1$, and 10 for (a)-(d) a twodimensional Gaussian initial distribution and (e) a narrow box distribution. All cases are evaluated without mean growth $(\bar{s}=0)$. Maxima of the joint PDFs decrease over time, and each contour curve decreases tenfold in magnitude [the value of the outermost contour of the final PDFs in (d),(e) is 0.001]. (a) A baseline case without fluctuations $\left(\sigma_{\mathrm{cf}}=0\right)$ for initial supersaturation distribution centered at $\varphi_{0}=1$ and $\alpha_{0}=1$, assuming $\xi=0.5$ and $\zeta=1$. (b) Effect of stronger damping of the supersaturation and slower depositional ice growth $(\xi=1, \zeta=0.5)$ and (c) effect of neglecting the growth due to an initial supersaturation. (d) As in (a), except including persistent supersaturation fluctuations ( $\sigma_{\mathrm{cf}}=0.1$ leading to $\sigma_{\infty}=0.05$ ). (e) As in (d), except using a very narrow initial box PDF. (f) Temporal evolution of supersaturation PDF obtained from (e) for $\theta=0$ (delta function), $0.5,1,2,5$, and 10 (asymptotic distribution in which the mean of the supersaturation fluctuations decayed to zero).

various cirrus cloud types. We distinguish between cirrus forming in situ—both in the midlatitudes (ML) and at the much colder tropical tropopause (TT) - and anvil cirrus (AV) detrained from deep convective cores. Furthermore, we include contrail cirrus (CC) as a special class of man-made upper-tropospheric ice clouds (Burkhardt and Kärcher 2011). We summarize observation-based parameters for each cirrus type used in this study in Table 1.

The table highlights the fact that damping and growth time scales vary considerably among different cirrus types. The ratio $\kappa$, signifying the relative roles of growth and damping, varies by almost three orders of magnitude, between 0.2 for young contrails (very strong 
TABLE 1. Summary of characteristic cloud-averaged parameters for individual cirrus types based on aircraft and remote sensing observations: total ice crystal number concentration $n$ and mean radii $r_{0}$, cloud temperature $T$ and pressure altitude $p$, areal growth time scale per unit supersaturation $\gamma^{-1}$ and damping time scale $\lambda^{-1}$, their ratio $\kappa=\gamma / \lambda$, and range of asymptotic size dispersion $\delta_{r}^{\infty}$ for weak to strong fluctuations. The supersaturation autocorrelation time $\tau$ used in the colored-noise model is set equal to 10 min to constrain the growth and damping parameters: $\zeta=\gamma \tau$ and $\xi=\lambda \tau$, respectively. Cloud types comprise tropical tropopause cirrus (TT; Peter et al. 2003; T. Peter 2013, personal communication; Jensen et al. 2013; E. Jensen 2013, personal communication), anvil cirrus (AV; Lawson et al. 2010; P. Lawson 2013, personal communication), midlatitude cirrus (ML; Field and Heymsfield 2003), and contrail cirrus (CC; Voigt et al. 2011; Minnis et al. 1998, 16 Apr case). The TT cases include cirrus with low (TT-L; Peter et al. 2003; T. Peter 2013, personal communication) and high (TT-H; Jensen et al. 2013; E. Jensen 2013, personal communication) ice crystal number densities based on in situ measurements. The AV cases represent many in situ observations of anvil cirrus attached (AV-A) and detached (AV-D) to tropical convection. The ML cases describe a single midlatitude cirrus cloud subdivided into regions predominantly containing many small (ML-S) and few large (ML-L) ice crystals; its properties have been estimated from cloud-resolving simulations of the observation case. The CC cases include in situ measurements of young ( $<10 \mathrm{~min}, \mathrm{CC}-\mathrm{Y}$; Voigt et al. 2011) and satellite observations of aged ( $\approx 7 \mathrm{~h}, \mathrm{CC}-\mathrm{A}$; Minnis et al. 1998, 16 Apr case) contrail cirrus, respectively. Mean ice crystal dimensions or effective radii inferred from the measurement data have been converted to approximate mean (volume-equivalent sphere) radii using relationships suitable for the most common ice crystal shapes in the respective cloud types as indicated by the measurements; the mean ice crystal radius in case TT-H is estimated based on measured total water. Measured microphysical properties of both anvil cases capture a range of cloud ages. The number concentration in case CC-A is only roughly estimated by scaling from CC-Y with an aircraft plume dilution factor of 0.01 . Data taken within each cirrus type (or within each individual cloud) may vary significantly, so care should be taken when generalizing the parameters assorted here as being typical. Citations refer to the original cloud observations.

\begin{tabular}{lcccccccccc}
\hline \hline Type & $n\left(\mathrm{~cm}^{-3}\right)$ & $r_{0}(\mu \mathrm{m})$ & $T(\mathrm{~K})$ & $p(\mathrm{hPa})$ & $\gamma^{-1}(\mathrm{~min})$ & $\lambda^{-1}(\mathrm{~min})$ & $\zeta$ & $\xi$ & $\kappa$ & $\delta_{r}^{\infty}$ \\
\hline TT-L & 0.005 & 5 & 190 & 100 & 4 & 370 & 2.5 & 0.03 & 93 & $0.4-0.5$ \\
TT-H & 0.3 & 6 & 190 & 100 & 5 & 6 & 2 & 1.7 & 1 & $0.085-0.25$ \\
AV-A & 1 & 35 & 230 & 250 & 22 & 0.45 & 0.5 & 22 & 0.03 & $\sim 0.01$ \\
AV-D & 0.1 & 20 & 220 & 250 & 1.5 & 8 & 7 & 1.2 & 6 & $0.3-0.4$ \\
ML-S & 0.05 & 30 & 228 & 320 & 2 & 14 & 5 & 0.7 & 7 & $0.3-0.4$ \\
ML-L & 0.002 & 110 & 239 & 390 & 7 & 100 & 1.4 & 0.1 & 14 & $0.35-0.4$ \\
CC-Y & 100 & 2 & 218 & 250 & 0.09 & 0.02 & 111 & 500 & 0.2 & $\sim 0.01$ \\
CC-A & 1 & 30 & 245 & 300 & 0.55 & 0.2 & 18 & 50 & 0.4 & $0.015-0.025$ \\
\hline
\end{tabular}

damping due to the high ice crystal number concentration and small mean size) and 93 for thin tropical tropopause cirrus (very weak damping due to small $n r_{0}$ values).

As cirrus clouds are spatially inhomogeneous and their physical properties change over time, we expect $\lambda$ and $\gamma$ to vary within a single cloud. To illustrate this point, we have performed detailed large-eddy simulations of case ML. In those numerical simulations, contrary to our present theoretical approach, the effects of production of ice due to nucleation; loss of ice due to complete sublimation, sedimentation, aggregation, turbulent mixing; and radiative-dynamical feedbacks are fully accounted for. We refer to Sölch and Kärcher (2011) for a detailed analysis of this observational case study.

Figure 3 depicts snapshots of the cloud's ice saturation ratio $(s+1)$ and ice water content (IWC) 1 and $3 \mathrm{~h}$ after formation, in order to demonstrate the tremendous degree of small-scale variability inside this cirrus. The water vapor mixing ratio inside the cloud (not shown) does not exhibit as much fine structure; it varies with altitude between 50 and $250 \mathrm{ppm}\left(0.02-0.14 \mathrm{mg} \mathrm{m}^{-3}\right)$ owing largely to the imposed vertical temperature profile [see Fig. 5 in Sölch and Kärcher (2011)] within a nonconvective atmosphere.

Although the simulated cirrus was forced by a moderate uplift, the large-eddy model created its own small-scale fluctuations in temperature and $\mathrm{H}_{2} \mathrm{O}$ mixing ratio, which is largely responsible for the fine structure seen in the simulated cloud fields. This self-induced model variability is generated by turbulence and waves, whose properties are affected by the limited computational domain. It is therefore very unlikely that it covers the full range of small-scale features that is typically present in nature (Fritts et al. 2013). In particular, water vapor variability would be larger in a convective background atmosphere (Fischer et al. 2012).

The $(s+1)$ field in the young cloud (Fig. 3a) reflects the rather smooth moisture profile used to initialize the simulations and is mainly determined by the imposed lapse rate. Above about $8-\mathrm{km}$ altitude, the air is ice supersaturated and subsaturated below. The entire cloud region is lifted at a wind speed of about $4 \mathrm{~cm} \mathrm{~s}^{-1}$, generating and maintaining supersaturation in the upper cloud region. Ice nucleation, growth, and sedimentation have already created some modulations in the $(s+1)$ field between 7.5 and $8.5 \mathrm{~km}$ (Fig. 3a), so ice crystals can form and grow there, eventually causing the fall streaks seen in Fig. 3c. Those modulations would be larger if the model contained more small-scale variability. Clearly, within the $2 \mathrm{~h}$ of cloud evolution, the spatial statistic of $(s+1)$ and IWC variability change markedly. 


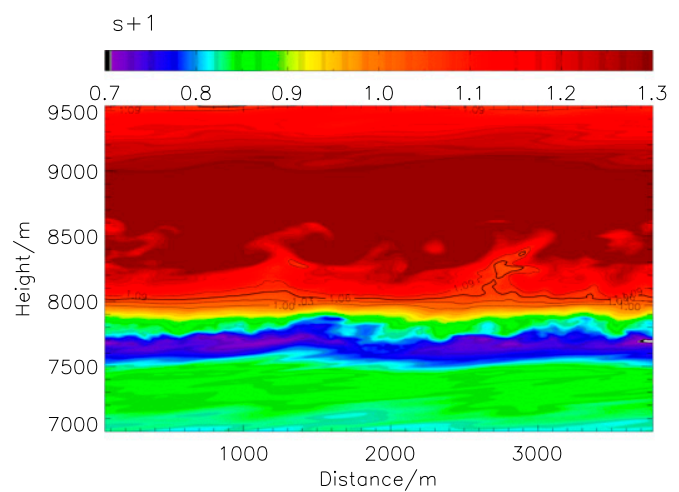

(a)

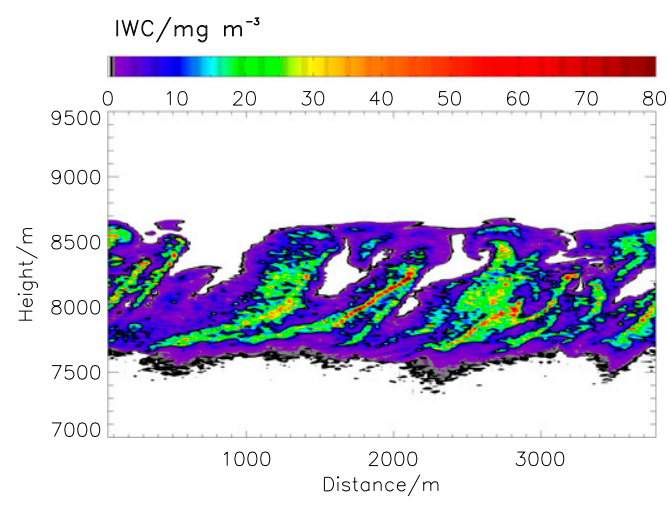

(c)

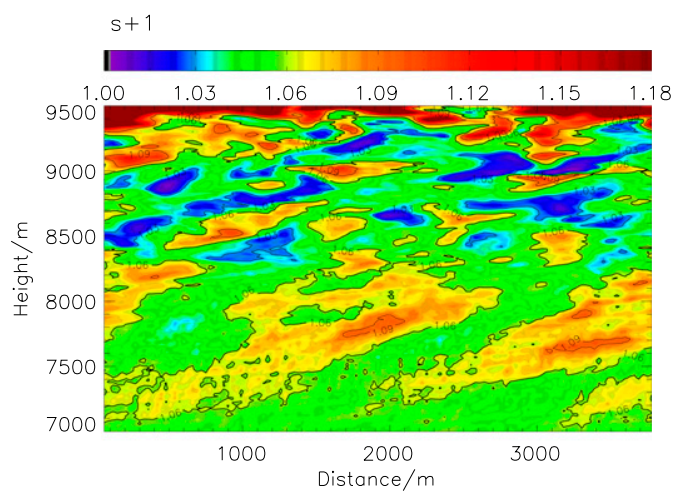

(b)

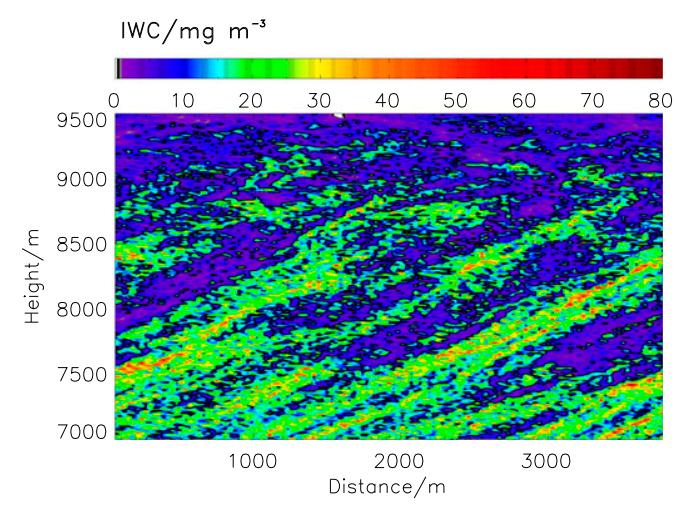

(d)

FIG. 3. Snapshots of (a),(b) ice saturation ratio and (c),(d) ice water content in a midlatitude cirrus cloud (case ML) taken from high-resolution large-eddy simulations [using 30 (20) m horizontal (vertical) resolution] about (a),(c) 1 and (b),(d) $3 \mathrm{~h}$ after in situ formation. According to observations, this regional cirrus cloud system was produced by slow uplift in air flowing northeasterly over mountainous terrain. The mature cloud extends over more than $3.5 \mathrm{~km}$ in the vertical. The simulations reveal a dramatic spatial variability of relative humidity and condensate, implying a similarly pronounced variability in damping and growth rates.

The $(s+1)$ field in the mature cloud (Fig. 3b) shows marked signatures of ice supersaturation quenching that correlate with the distribution of ice crystals, as expected from our idealized theoretical approach. Patches of $(s+1)$ values higher and lower than the domain-average value $(\simeq 1.06)$ alternate; the quenching effect appears to be weaker in the lower cloud region, where ice crystal number concentrations are very small. Furthermore, the few individual fall streaks seen in the young clouds' IWC field have been replaced by many filamentary structures $2 \mathrm{~h}$ later (Fig. 3d).

To connect such information to our theory, parameters were calculated in grid cells containing cirrus ice crystals using local conditions; that is, $\lambda$ was calculated according to Eq. (9) and $\gamma$ was calculated using grid cell temperatures and mean (volume-equivalent sphere) radii. By virtue of the large-eddy simulation results, we are able to distinguish two ML cloud regions: ML-S and ML-L. We define that ice crystals with number concentrations larger (smaller) than $0.01 \mathrm{~cm}^{-3}$ belong to the ML-S (ML-L) category. The ML-S region evolves between 8.2 and $8.5 \mathrm{~km}(301-339 \mathrm{hPa})$ and is characterized by temperatures $224-232 \mathrm{~K}$, ice saturation ratios $1.06-1.49$, and mean ice crystal radii $11-49 \mu \mathrm{m}$. The ML-L region evolves between 6 and $7.5 \mathrm{~km}$ (357$417 \mathrm{hPa}$ ) and comprises the ranges $235-243 \mathrm{~K}, 0.8-0.95$, and $102-115 \mu \mathrm{m}$, respectively. ML-S is highly supersaturated over ice most of the time, representing the upper cloud region where many ice crystals nucleate and undergo rapid depositional growth. ML-L is frequently subsaturated, comprising the sublimation zone at low levels; the fewer ice crystals there enter from ML-S by sedimentation.

Figure 4a shows the simulated fractions of supersaturated cloudy air within the ML-S and ML-L regions versus time. These fractions are defined as the volume of grid cells that contain ice crystals and in which $s>0$, divided by the total cloud volume in the respective 

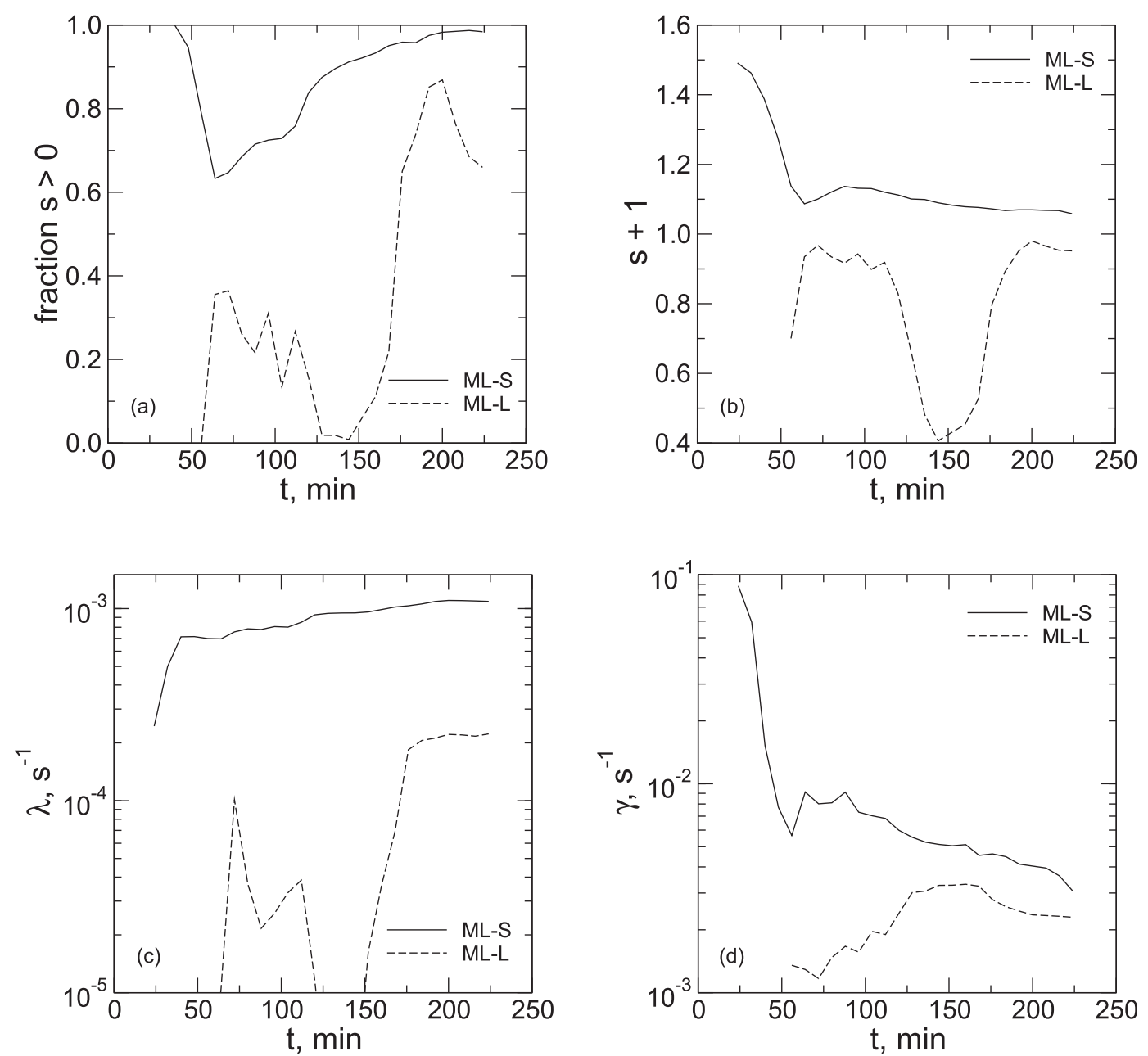

FIG. 4. (a) Simulated temporal evolution of the fraction of cloudy air that is supersaturated, obtained from cloudresolving simulations of a midlatitude cirrus cloud (Fig. 3) by averaging $s$ over the upper (solid curve) and lower (dashed curve) cloud regions representing small and large mode ice crystals (ML-S and ML-L, respectively). Time evolution of the (b) average ice saturation ratio and (c) damping and (d) growth rates in both regions. Ice crystals nucleate in the upper cloud region only after $30 \mathrm{~min}$. Data in ML-L start later than in ML-S since the ice crystals need to settle to lower levels first; the time required for cloud variables in ML-L to be properly defined is about $1 \mathrm{~h}$.

region. The evolution of the ice saturation ratio, averaged in both regions, is presented in Fig. 4b. In the simulations, ice crystals form by homogeneous freezing of supercooled aerosols when the ice saturation ratio exceeds approximately 1.45 .

By means of Fig. 4, we also discuss the evolution of Fig. $4 \mathrm{c} \lambda$ and Fig. 4d $\gamma$ in volumes containing many small (ML-S) and few large (ML-L) ice crystals separately. The damping rate $\lambda$ increases with time and reaches an almost constant value after $150 \mathrm{~min}$ of cloud evolution in both regions. The final values differ significantly, by about a factor of 7 . The initial increase in $\lambda$ in the ML-S region is caused by the nucleation and depositional growth of ice crystals, while microphysical sources and sinks of ice crystals come into approximate balance at later stages, keeping $\lambda$ constant. In the ML-L region, $\lambda$ increases initially by settling of ice crystals from ML-S, decreases between 50 and 100 min owing to enhanced sublimation, and increases again as soon as larger cloud portions become supersaturated.

The growth rate $\gamma$ is fairly constant in ML-L, because temperature and ice crystal surface area exhibit only small variations. This indicates that the population of sedimenting ice crystals has rather uniform sizes and that depositional growth of such large ice crystals is inefficient. By contrast, $\gamma$ from ML-S decreases rapidly as the cloud ages. The high initial $\gamma$ values are caused by cold temperatures and small ice crystal sizes (shortly after nucleation) and approach those from ML-L, as ice 
crystals grow and begin to settle into warmer (lower) cloud regions.

The marked internal variability in damping and growth conditions is clearly not limited to the ML case studied here. Observations of the highly variable microphysical structure of many upper-tropospheric cirrus (Comstock et al. 2004) and anvil cirrus (Lawson et al. 2010) underscore that nonequilibrium regions exist within other cirrus types as well.

\section{c. Ice crystal size dispersion}

Returning to our idealized stochastic model, we define the relative size dispersion as $\delta_{r}=\sqrt{\sigma_{r}^{2}} / r_{m}$, using the variance and mean radius of the radial size PDF:

$$
\sigma_{r}^{2}(\theta)=\int_{0}^{\infty}\left(r-r_{m}\right)^{2} P_{r} d r, \quad r_{m}=\int_{0}^{\infty} r P_{r} d r .
$$

The dispersion may be viewed as a heterogeneity parameter quantifying variability: large (small) values indicate heterogeneous, broad (homogeneous, narrow) distributions. We make use of $\delta_{r}$ in order to examine relationships between fluctuations of supersaturation and the resulting variability in ice crystal sizes.

For the remainder of this study, we define the initial distribution in $\varphi$ as a narrow top hat. Numerically, this function is approximated by $1 / \Delta \varphi$, with the grid increment $\Delta \varphi$. Furthermore, we choose a very narrow tophat initial PDF in radius space as well such that the initial value of $\delta_{r}$ is 0.01 . All simulations are again carried out using $\varphi_{0}=0$. This means that changes in size space are solely caused by supersaturation variability. We assign a constant value, $\tau=10 \mathrm{~min}$, to the Lagrangian supersaturation autocorrelation time in order to fix the dimensionless damping and growth factors $\xi$ and $\zeta$ (section $2 \mathrm{~b}$ ). We simulate size dispersions using two values of the undamped supersaturation fluctuation amplitude, $\sigma_{\mathrm{cf}}=0.05$ and 0.15 representing weak and strong forcing, respectively.

Since small ice crystals respond to changes in $\varphi$ faster than large ones, we may expect PSDs to broaden in the presence of supersaturation fluctuations (Kärcher 2012). Broadening will be most pronounced during sublimation phases $(\varphi<0)$, in which ice crystals shrink very rapidly. In all cloud cases, size dispersions increase over time to reach a steady state. Asymptotic values represent stationary conditions, where the fluctuation variance approached its limiting, damped value [Eq. (B8)] and where the effect of fluctuations and changes in ice crystal size balance each other. The data compiled in Table 1 give the ranges of asymptotic values $\delta_{r}^{\infty}$ (taken at $\theta=10$ ), resulting from the above range for $\sigma_{\mathrm{cf}}$.
Our results suggest a damping regime-oriented classification of cirrus, describing the ability of supersaturation variability to affect PSDs-hence, the development and radiative response of cirrus. We find the greatest contrast of $\delta_{r}^{\infty}$ between the cases CC-Y and TT-L with very many (strong damping) and extremely few (weak damping) ice crystals, respectively. Anvil clouds attached to convection are another example for strongly damped cirrus, while detached anvils and midlatitude cirrus can be considered more weakly damped clouds. Contrail cirrus happens to be strongly damped as long as $n>1 \mathrm{~cm}^{-3}$. Similar to cases TT, the two AV cases exhibit a large contrast in damping rates within a single cirrus type. Inasmuch as the parameters assigned to each cirrus type can be regarded typical for the cloud as a whole, weak (strong) damping consistent with $\kappa>1$ $(<1)$ leads to large (small) PSD dispersions. However, the discussion of the ML case in section $3 b$ reminds us that, despite this cloud-average regime classification, a range of local damping rates is likely to be present within single clouds in all cirrus types.

Recalling Fig. 2f, we suspect that transient supersaturation should often be found in TT and ML clouds while present only in thin portions (cloud edges, fall streak regions) of AV and CC clouds. We study the TT cases in more detail in section 4 , but we do not discuss the ML cases any further, since ice crystals with large sizes sediment quite rapidly and our model can no longer be applied realistically.

In weakly damped cloud regions, supersaturation fluctuations may lead to nucleation of ice crystals, whereas ice is very unlikely to form in strongly damped regions, even when fluctuation amplitudes are relatively high. Our findings do not necessarily imply that PSDs should always be narrow in (portions of) strongly damped cirrus. For instance, contrails and anvils are characterized by enhanced turbulence kinetic energy relative to upper-tropospheric background values. Enhanced turbulent mixing causes air parcels with different particle growth histories to mix more rapidly, which can broaden PSDs compared to those to those evolving within individual parcels, while the PDF of supersaturation could still be very narrow. In contrast, the laminar appearance of thin cirrus at the tropical tropopause (Winker and Trepte 1998) hints at comparatively weak or slow interparcel mixing; this might indicate that the small-scale dynamical forcing can play a more important role in shaping PSDs in those clouds than in most other cirrus types.

\section{Tropical tropopause cirrus}

The occurrence and distribution of cirrus in the tropical tropopause layer (above 15-km altitude) is closely 
coupled to large-scale tropical circulations (Randel and Jensen 2013). Our results suggest that there is a smallscale microphysical control of ice supersaturation, which affects the degree of supersaturation and ice mass present in TT clouds, hence the efficacy of freeze drying as air passes through the tropical tropopause. Exactly to what degree dehydration is overestimated in water vapor trajectory calculations frequently employing saturation adjustment ${ }^{3}$ (Fueglistaler et al. 2005; Liu et al. 2010) remains to be properly quantified, as microphysical properties, and therefore the quenching of supersaturation, can vary greatly among individual TT cirrus clouds (Jensen et al. 2013).

The two distinct TT cirrus cases listed in Table 1 cover observed types of ice clouds that are closest to the very cold tropical tropopause, providing the last contact of air with ice crystals-the final possibility for freeze drying-before it enters the much drier stratosphere. Observations suggest a separation of TT cirrus into in situ-generated cirrus (modulated by the planetary-scale vertical velocity field) and cirrus derived from spreading anvils of convective clouds (showing a different timevarying spatial pattern of cloud fraction) (Virts et al. 2010), likely with different microphysical properties (Wang and Dessler 2012). The highest TT cirrus clouds presumably form in situ, for instance in the cold phases of Kelvin waves with periods of several days, and can persist for several days (Jensen et al. 1996; Dinh et al. 2010). Anvils detrained from deep convective outflow are prevalent at lower altitudes $(12-14 \mathrm{~km})$ and are also characterized by long, albeit highly variable, lifetimes (Luo and Rossow 2004; Feng et al. 2012).

Air enters the stratosphere mainly in the tropics via slow, diabatic ascent across the tropopause (Holton and Gettelman 2001). Because of their important role in regulating stratospheric humidity, we study supersaturationgrowth interactions within TT cirrus in more detail and relate our findings to the ability of falling ice crystals to irreversibly remove total water from the tropical tropopause layer. Owing to their small settling velocities, only the smallest ice crystals penetrate the tropopause and eventually sublimate, thereby adding their water content to stratospheric air. Terminal fall speeds of the largest ice crystals present in TT clouds can exceed $1 \mathrm{~cm} \mathrm{~s}^{-1}$, which is about 10 times larger than mean (large scale) vertical wind velocities balancing radiative heating in the tropical tropopause layer. Therefore, a requirement for efficient dehydration induced by sedimenting cloud ice in TT cirrus is met, in which case the effectiveness of freeze

\footnotetext{
${ }^{3}$ Any water vapor above the saturation is regarded as precipitable condensate and immediately removed from the trajectory.
}

drying depends crucially on the number concentration of the largest ice crystals (i.e., on the shape of the PSD that is affected by small-scale supersaturation variability).

A crucial quantity controlling potential dehydration effects of TT cirrus is the water mass flux due to ice crystal sedimentation, $\Phi=\int_{0}^{\infty} v_{t} \mathrm{IWC} P_{r} d r$. With the Stokes terminal fall velocity for spherical particles, $v_{t}=$ $2 g \varrho r^{2} /(9 \eta)$, and the ice water content, IWC $=4 \pi \varrho n r^{3} / 3$, where $\eta(T)$ is the dynamic viscosity of air, $\varrho(T)$ is the mass density of bulk ice, and $g$ is the acceleration of gravity, we formulate the following relationship for the sedimentation ice mass flux:

$$
\Phi(\theta)=\frac{8 \pi g Q^{2} n r_{0}^{5}}{27 \eta} \underbrace{\int_{0}^{\infty}\left(\frac{r}{r_{0}}\right)^{5} P_{r} d r}_{\equiv M_{5}} \propto n r_{0}^{5} M_{5} .
$$

Being proportional to the fifth moment of $P_{r}, \Phi$ is very sensitive to the abundance of large ice crystals.

Tropical tropopause cirrus with very low total ice crystal number concentrations (case TT-L) have been observed during the Airborne Polar ExperimentThird European Stratospheric Experiment on Ozone (APE-THESEO) field campaign (Peter et al. 2003). According to in situ and lidar measurements, those clouds appear to be rather homogeneous horizontally, covering areas up to several $1000 \mathrm{~km}^{2}$ and occur very frequently in the tropics. They are extremely tenuous, with mean radii of 5-6 $\mu \mathrm{m}$, ice crystal number concentrations $(>3 \mu \mathrm{m})$ of $0.005-0.01 \mathrm{~cm}^{-3}$, and with only $1 \%-$ $5 \%$ of the total available water residing in the condensed phase (corresponding to $\mathrm{H}_{2} \mathrm{O}$ mixing ratios of 0.025 $0.1 \mathrm{ppm})$. While formation processes are not known, a delicate stabilization mechanism is required in order to explain their longevity (Luo et al. 2003). In the more recent Airborne Tropical Tropopause Experiment (ATTREX), Jensen et al. (2013) reported airborne cirrus measurements in similar ambient conditions but with much higher total number concentrations (TT-H). Those clouds do not seem to occur very frequently. Low number concentration cirrus have also been probed during ATTREX, but much less is known as to their origin and development.

Using the parameters listed in Table 1 to constrain simulations of TT-L and TT-H clouds, we show in Fig. 5 the evolution of their radial PDFs, $P_{r}$, at selected times after onset of colored noise. As before, we evaluate both cases using very narrow initial $P_{r}$, centered at $r_{0}=$ 5 (6) $\mu \mathrm{m}$ in case TT-L in Fig. 5a (TT-H in Fig. 5b). The PDFs at later times are solely created by rapid supersaturation fluctuations. As before, we prescribe $\tau=$ $10 \mathrm{~min}$ and $\sigma_{\mathrm{cf}}=0.05 ; 0.15$. With these choices, the 

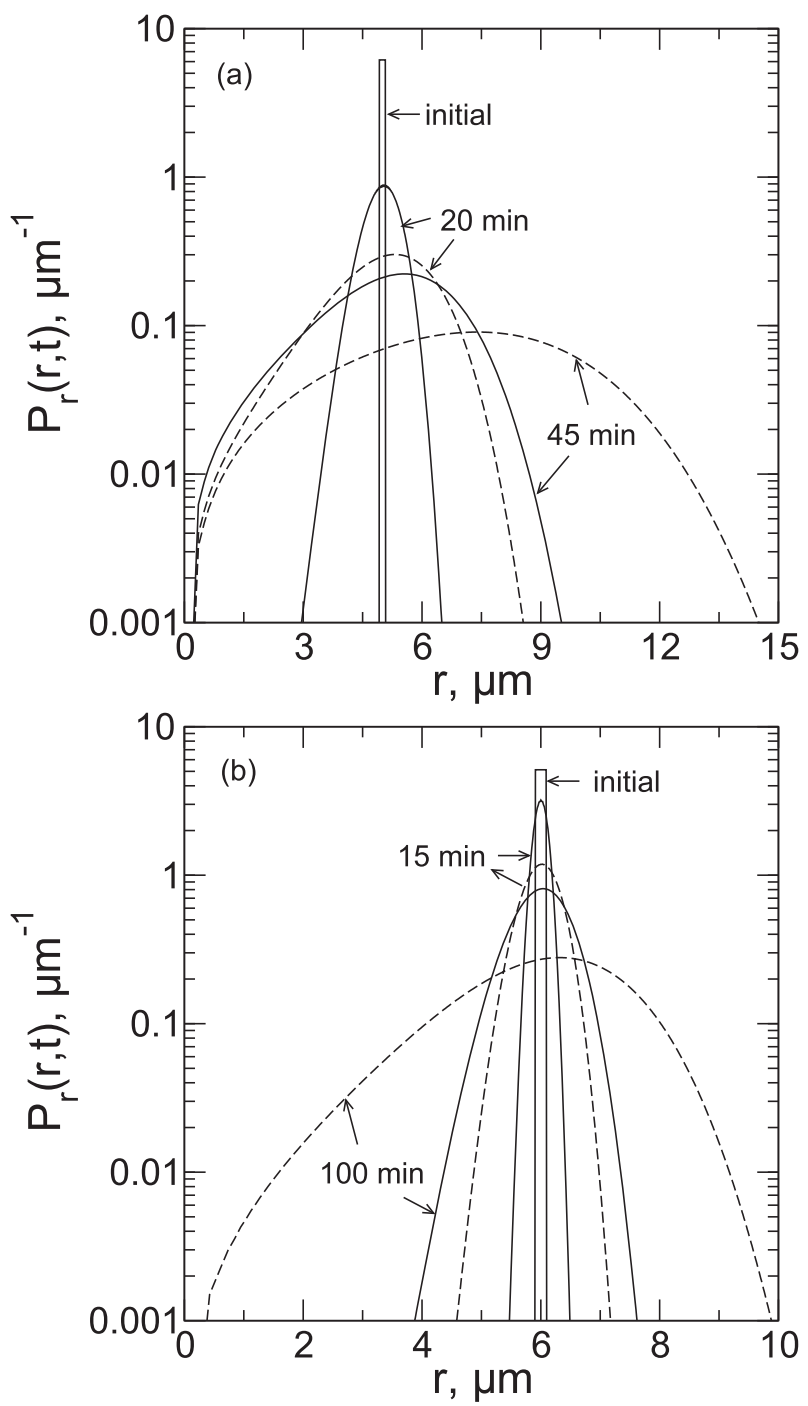

FIG. 5. Simulated ice crystal size PDFs at different times for (a) TT-L and (b) TT-H cirrus. Solid (dashed) curves are computed assuming small (large) supersaturation fluctuation amplitudes. Labels denote the times after onset of the fluctuations acting on an initially monodisperse ice crystal population at ice-saturated conditions. Ice crystals sublimate as $r \rightarrow 0$.

damped (in cloud) asymptotic supersaturation fluctuation amplitudes are $\sigma_{\infty}=0.02,0.05$ for TT-H and $\sigma_{\infty}=$ 0.04; 0.6 for TT-L. The latter value happens to be unrealistically large and may not be maintained over more than a few minutes' time, suggesting a missing process in determining the damping rate $\lambda$ used in our model. Such an additional damping mechanism is likely tied to fluiddynamical instabilities generated at high fluctuation amplitudes that must ultimately lead to turbulent and molecular dissipation of the fluctuation energy.

Starting with top-hat distributions, the PDFs broaden and become asymmetric (e.g., see the left-skewed PDFs at $t=20 \mathrm{~min}$ in TT-L; Fig. 5a), since smaller crystals respond to the fluctuations faster than larger ones. As expected, the size broadening is more pronounced in case TT-L relative to TT-H (Fig. 5b) owing to the lower damping rate. In both cases, the broadening effect increases with stronger forcing, compare solid and dashed curves. As the ice crystal populations are initially saturated and experience positive and negative $\varphi$ values, some of the smallest crystals completely sublimate over time at the lower radial boundary. This starts to become relevant at $100 \mathrm{~min}$ in case TT-H but already after $20 \mathrm{~min}$ in case TT-L. As detailed in section 2a, the source of water molecules due to completely sublimated ice crystals is not accounted for in our model. We limit our analysis to times after onset of the fluctuations where $\iint P d \varphi d \alpha>0.8-0.9$ (i.e., we tolerate an error of at most $10 \%-20 \%$ in the conservation of total water in our model in aged TT cirrus).

That said, in the ensemble of air parcels experiencing different realizations of random supersaturation fluctuations, there will always be a few ice crystals that preferentially encounter most favorable growth conditions, enabling them to reach radii much larger than $r_{0}$. Therefore, supersaturation fluctuations may establish an in situ route to creating very few very large ice crystals without the need to impose a mean supersaturation, which would otherwise be difficult to explain. Indeed, aircraft observations near the tropical cold point identified less than $10^{-5} \mathrm{~cm}^{-3}$ ice crystals as hexagonal plates with aspect ratios of 6:1 and maximum lengths of $100 \mu \mathrm{m}$ (Jensen et al. 2008). Such particles correspond to volumeequivalent spheres with $r \approx 36 \mu \mathrm{m}$.

To conclude the discussion, we make an attempt to assess the realism of the simulated size PDFs by comparison to in situ measurements for case TT-L, using the data from Table 1 as constraints and assuming weak forcing $\left(\sigma_{\mathrm{cf}}=0.05\right)$. To relate our model results directly to PSDs in the form $n r P_{r}$ taken during APE-THESEO [cf. Fig. 5 in Peter et al. (2003)], we present in Fig. 6 the model curves without symbols taken 15 (dashed) and $40 \mathrm{~min}$ (solid) after the onset of the fluctuation source acting on the initially very narrow size distribution. The three sets of measurements are shown as curves with symbols; these data suggest large PSD dispersions, similar to the asymptotic values $\delta_{r}^{\infty}$ from Table 1 .

Since the observations provide no clue on the history and age of the cirrus and its dynamical forcing, we may regard the comparison as encouraging. The simulation result at $15 \mathrm{~min}$ is still affected by the assumption of a monodisperse initial PSD and the agreement with the data could be improved by fitting a broader PSD as the initial distribution. Likewise, the agreement between the result at $40 \mathrm{~min}$ and the data could be improved 


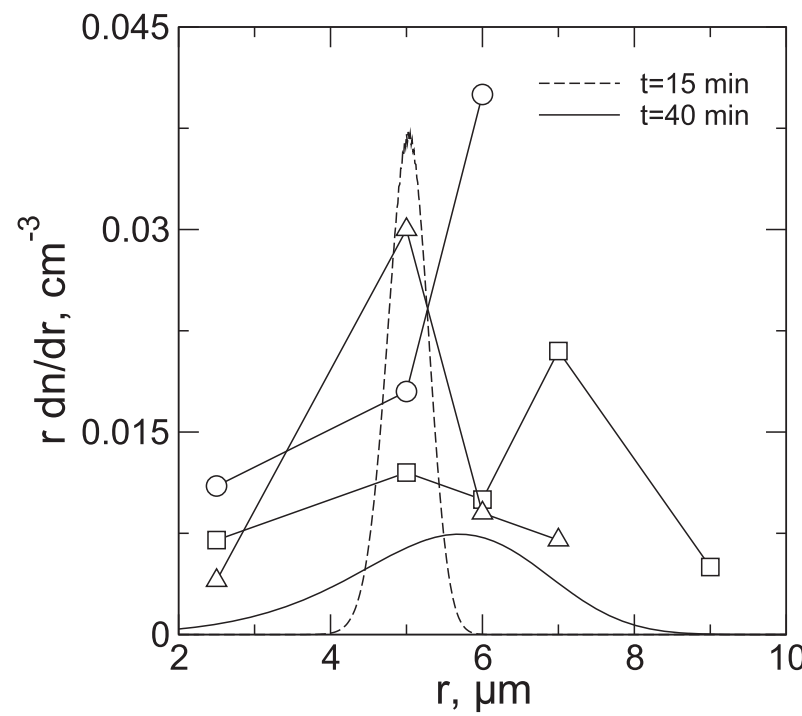

FIG. 6. Ice crystal size distributions vs volume-equivalent sphere radius from the APE-THESEO campaign (Peter et al. 2003) (curves with symbols, connected with lines to guide the eye) and from our model for TT-L cirrus subject to weak supersaturation fluctuations. The two curves without symbols are model results taken at times $t=15$ (dashed, $\theta=1.5$ ) and $t=40 \mathrm{~min}$ (solid, $\theta=4$ ) after onset of supersaturation variability. The initial distribution is shown in Fig. 5a.

by fitting the fluctuation source strength and selecting a proper cloud age. Because of the lack of more detailed experimental information and the difficulty in sizing ice crystals using optical spectrometer probes, a more detailed comparison and further interpretation is not attempted.

Supersaturation fluctuations have also implications for the water mass flux $\Phi$ because of sedimentation. As both TT cases are characterized by very similar air temperatures and pressures, differences in $\Phi$ according to Eq. (14) are brought about by differences in $n r_{0}^{5}$ and the specific features of the ice crystal PDF, determining $M_{5}$ shown in Fig. 7 as a function of the scaled damping factor $\xi$ and corresponding supersaturation quenching times $\lambda^{-1}$. All simulations assume $\zeta=2$ and interpolate between TT-L and TT-H based on their ability to dampen the imposed fluctuations and are performed for weak (circles) and strong (squares) forcing. The M5values have been extracted from the simulations at various times after onset of the fluctuations to avoid situations with excessive sublimation; open symbols are taken in the asymptotic regime $\left(\theta=9-10, \iint P d \varphi d \alpha=\right.$ $0.9-1)$, while filled symbols are taken at intermediate times $\left(\theta=4-5, \iint P d \varphi d \alpha=0.8-0.9\right)$. The latter cases underestimate the true M5 values, since our model becomes inaccurate when the sublimation sink becomes significant (in case of weak damping, $\xi<0.2$ ). The

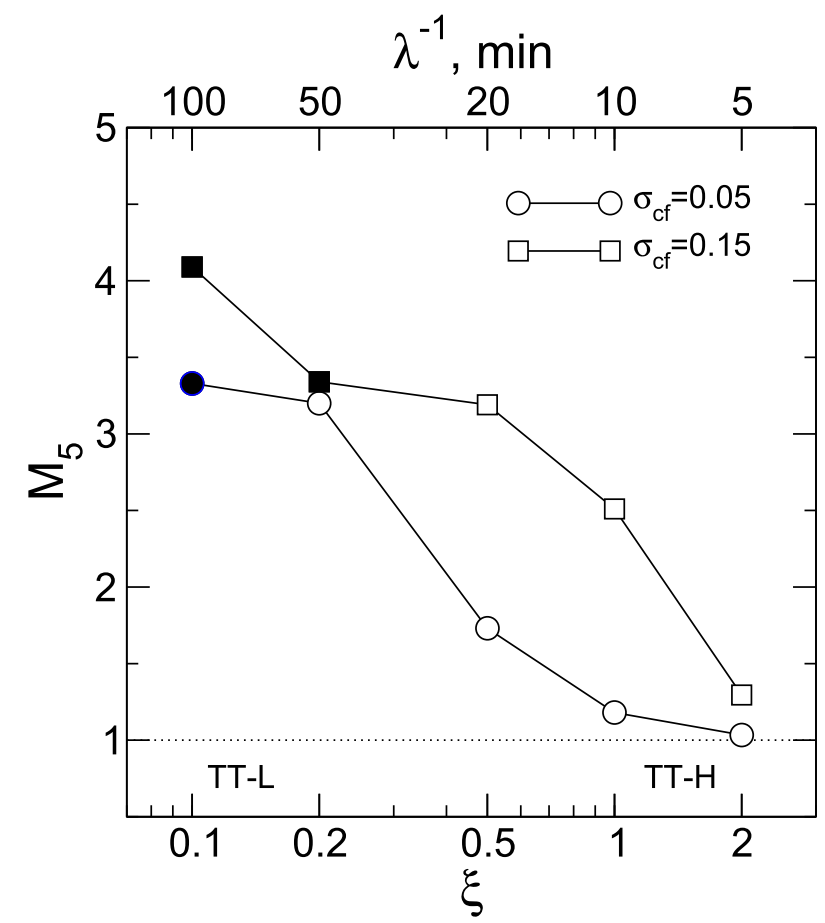

FIG. 7. Normalized water flux due to ice crystal sedimentation in tropical tropopause cirrus vs damping factor (bottom axis) or equivalent supersaturation relaxation time (top axis). Circles (squares) assume weak (strong) forcing and represent different times after onset of supersaturation variability; filled symbols are lower-limit values (see text). The simulations interpolate between low and high ice crystal number concentration TT cirrus in terms of the ability of these clouds to dampen the fluctuations by ice growth.

underestimation is larger for stronger forcing, because besides more sublimation, the minimum and maximum fluctuation amplitudes partly fall outside physical limits at large $\theta$; this supports our conjecture that we lack an additional (dynamically induced) damping process that would set a lower-limit value for $\xi$.

While $M_{5}$ remains close to its initial value of unity in more strongly damped TT cirrus $(\xi>1)$, PSD broadening and ice crystal growth cause $M_{5}$ to increase considerably with decreasing damping strength, reaching values greater than 3 , depending on the amplitude of the fluctuations. We conclude that size broadening is an important mechanism to increase the mass flux in TT cirrus with relatively low ice crystal number concentrations $\left(<0.1 \mathrm{~cm}^{-3}\right)$. However, since $n r_{0}^{5}$ in strongly damped cirrus can be orders of magnitude smaller than in weakly damped cirrus, the former still cause a larger vertical ice water flux. $\mathrm{H}_{2} \mathrm{O}$ vapor in the air within TT-H cirrus is close to saturation owing to fast supersaturation quenching times $(<10-20 \mathrm{~min})$. Another interesting finding is that any supersaturation in TT-L cirrus can be maintained over a period of at least $1 \mathrm{~h}$. This is 
consistent with in situ measurements of frequent ice supersaturation in the upper tropical troposphere, provided temperatures are low enough to ensure an already elevated background relative humidity near ice saturation and that small-scale variability in supersaturation is ubiquitous.

Our analysis assumes that ice crystals possess a hexagonal lattice structure, as we use the ice saturation vapor pressure for this most common type of ice. However, it is likely that cirrus particles nucleate preferentially in the form of cubic ice crystals at low temperatures (Kuhs et al. 2012) and only slowly transform into the thermodynamically more stable hexagonal crystals. This transformation may alter PSDs through a Bergeron-Findeisen-like process (Murphy 2003), potentially enhancing ice mass fluxes. At this level of detail, also radiative-dynamical interactions, leading to prolonged maintenance of cirrus against sedimentation (Durran et al. 2009), the shape of the falling ice crystals, determining their fall speeds and growth rates (Jensen et al. 2008), and kinetic limitations of depositional growth of ice crystals (Zhang and Harrington 2014) become important. The limited set of experimental information on TT cirrus and the difficulty in measuring their microphysical properties, in particular, PSDs and ice crystal shapes (Lawson et al. 2008; Davis et al. 2010), hampers accurate consideration of those factors in theoretical studies.

\section{Conclusions}

\section{a. Summary}

We have discussed the occurrence of small-scale supersaturation fluctuations within cirrus clouds and their effect on ice crystal size distributions. We have devised a theoretical framework integrating a minimal set of physical processes, small-scale variability in ice supersaturation and ice crystal growth by uptake of water vapor, required to study the problem delineated here with a probabilistic model. It may serve as a starting point for more detailed theoretical or numerical investigations. As a first important application, we have examined the microphysical potential of cold tropical tropopause cirrus to exhibit thermodynamic nonequilibrium states and to irreversibly remove water from the cloud layer.

Our work highlights the importance of distinguishing between various cirrus cloud types in terms of their ability to quench (dampen) supersaturation fluctuations. We suggest distinguishing between weakly damped and strongly damped ice clouds for scientific study. Various degrees of departures from ice saturation can occur in cirrus, depending primarily on the total number and mean size of cirrus ice crystals and the intensity of the fluctuation source (dynamical forcing). Typically, supersaturation will occur within many tropical tropopause and midlatitude cirrus. On the other hand, anvils and contrail cirrus more typically exhibit strong damping and therefore much less frequent occurrence of nonequilibrium states. We have illustrated, by means of large-eddy simulations of a midlatitude ice cloud, that marked spatial and temporal variations of damping conditions can also occur within single clouds owing to their spatial and temporal heterogeneity. Other cirrus types exhibiting large internal variability in water vapor and cloud ice are known to exist, and the ties between observed in-cloud supersaturation and dynamical forcing as the primary cause of nonequilibrium states remain to be examined in greater detail.

Our primary findings are summarized as follows:

1) Deviations from ice saturation in cirrus are small except in weakly damped cirrus, where supersaturation fluctuations broaden ice crystal size distributions. Broad ice crystal size distributions in strongly damped cirrus are likely caused by spatial mixing.

2) Significant supersaturation, and potentially new ice formation, only occurs in regions with few small ice crystals, depending on the variance of small-scale supersaturation fluctuations.

3) In weakly damped tropical tropopause cirrus, water mass fluxes due to sedimentation increase significantly, ice supersaturation persists, and few large ice crystals form in the presence of supersaturation fluctuations.

\section{b. Implications}

Various authors have pointed out links between largescale processes and supersaturation variability in cirrus. While such links exist-determining the synoptic-scale macrophysical properties (e.g., frequency of cloud occurrence) and the overall evolution of cloud microphysics (e.g., ice water content)—our study underscores the strong microphysical control of supersaturation that may counteract even rather vigorous dynamical forcing. This microphysical supersaturation quenching mechanism enables a significant amount of supersaturation to occur only in (portions of) cirrus with few small ice crystals. A major candidate of such nonequilibrium clouds with serious climatic implications is thin cold cirrus, developing in the tropics above the mean level of convective outflow.

Disparate types of tropical tropopause cirrus with low and high ice crystal number concentrations have been identified in airborne measurements, but it is difficult to ascertain which type is more representative throughout the tropics. It is therefore of paramount 
importance to better characterize ice nucleation processes that eventually determine the total ice crystal concentration, since cirrus with different particle number will develop very differently. Ice nucleation rate coefficients are sensitive functions of relative humidity and temperature; hence, ice crystal formation will also be controlled by small-scale variability in supersaturation. Our findings imply that preexisting ice crystals will often affect the ice formation process as well via their ability to dampen supersaturation fluctuations.

We have identified several interesting effects in lownumber-concentration tropical cirrus that might be helpful in better assessing the role of these clouds in controlling stratospheric water vapor and hence climate, as well as in interpreting field measurements. Vertical redistribution of water due to sedimentation that is enhanced by ice crystal size spreading and long-lived supersaturated states-both induced by small-scale supersaturation fluctuations-make it more difficult to estimate their freeze-drying potential (e.g., in dynamical trajectory calculations that do not explicitly simulate small-scale cloud processes). Frequent observations of supersaturated states in airborne tropical measurements are consistent with the quasi-persistent nature of supersaturation in tropopause cirrus with low damping rates, as discussed here. Furthermore, supersaturation variability is likely to be a major controlling factor of ice formation in aerosol particles with disparate ice nucleation efficiencies. Therefore, supersaturation fluctuations might alter the susceptibility of these cirrus clouds to heterogeneous ice nuclei. Lack of representing supersaturation variability in models compromises the fidelity with which moisture transport into the stratosphere can be simulated.

The fact that the fine structure of supersaturation variability in cirrus is virtually unconstrained by observations renders the development of a rigorous physical theory on which cirrus modeling could be based difficult. Nonetheless, we expect to arrive at similar conclusions with other noise sources, provided that the total power of the supersaturation fluctuations is similar. It is therefore necessary to (i) estimate supersaturation variances in cirrus and compare them with those predicted by the colored-noise model and (ii) identify dynamically induced damping processes that add to the diminishing microphysical damping in low-numberconcentration cirrus and are likely to be highly variable in nature. Furthermore, it needs to be studied whether other stochastic approaches (e.g., models allowing for nonstationarity and intermittency of supersaturation fluctuations) are better suited to describe the problem at hand. Measuring frequency power spectra and structure functions of atmospheric supersaturation fluctuations in cirrus could be a viable way to constrain stochastic models. We therefore postpone a discussion of the quantitative accuracy of our results based on a damped colored-noise source until more basic information becomes available.

Sufficiently strong turbulent mixing driven by spatial gradients of cloud variables can also lead to a broadening of ice crystal size distributions. The effect of mixing, particularly in cirrus with enhanced turbulence kinetic energies, warrants further study and necessitates the use of a comprehensive, high-resolution cloud model. In such numerical models, it is a challenge to properly represent supersaturation fluctuations, since the model-generated noise may not be similar to the natural variability.

The cirrus features described here will be difficult to capture in aircraft measurements. The high speed of airborne platforms and the limited time resolution of most instruments likely lead to a lack of temporal and spatial context in which, for example, measured and modeled (joint) PDFs of cirrus properties could be compared. Therefore, a challenge in designing future cirrus measurements will be to identify those variables that are most easily comparable-and at the same time most meaningful-to the present type of modeling. Furthermore, we point out that a direct comparison of deterministic cloud models with single observational case studies can be misleading. Instead, the stochastic nature of small-scale dynamical ice cloud forcing calls for comparisons with cloud-averaged values or for ensemble simulations.

Finally, investigations of two-way interactions between cloud-scale processes and the synoptic-scale forcings determining the confines in which cirrus form, evolve, and dissipate hold rich opportunities for future research into cirrus clouds.

Acknowledgments. Preliminary results of this study were presented and discussed at the 535. WE-Heraeus Seminar "Water Vapor and Ice in the Atmosphere" (Bad Honnef, Germany, July 2013) hosted by the German Physical Society (DPG). We thank Ulrike Burkhardt, Eric Jensen, Beiping Luo, Thomas Peter, Markus Rapp, and Mark Zondlo for fruitful discussions; Paul Lawson, Sara Lance, and Qixu Mo for providing us with mean ice crystal sizes from microphysical data taken in anvil cirrus during the NASA TC4 field campaign; and Anton Bruckner for encouragement while conducting this research. This work is a contribution to the research program "High Definition Clouds and Precipitation for advancing Climate Prediction" (HD $(\mathrm{CP})^{2}$ ), directed by Bjorn Stevens and funded by the 


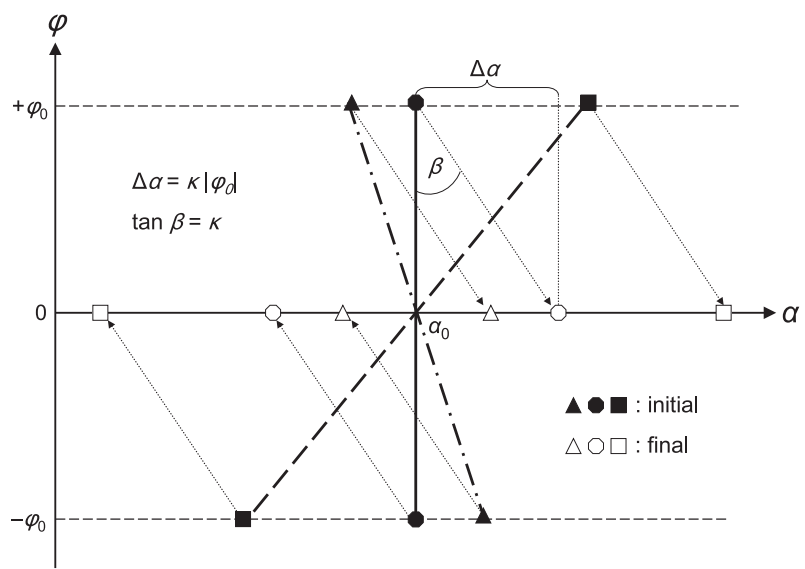

FIG. A1. Schematic illustrating the evolution of single-ice-crystal trajectories in $\{\alpha, \varphi\}$ phase space in the absence of supersaturation fluctuations. Filled and open symbols indicate initial and final conditions, respectively. Large $\kappa$ values induce large angles $\beta$, indicating that growth is fast relative to damping. Damping (i.e., quenching of the initial supersaturation) occurs because of both vapor deposition $(\varphi>0)$ and sublimation $(\varphi<0)$.

Federal Ministry of Education and Research in Germany (BMBF).

\section{APPENDIX A}

\section{Unforced Phase-Space Solution}

We derive the single-particle phase-space trajectories $\alpha(\varphi)$ neglecting the random forcing $(\epsilon=0)$. Solving Eq. (3) for a vanishing ensemble-mean supersaturation and inserting the solution into Eq. (2) yields in dimensionless variables

$$
\begin{aligned}
& \varphi(\theta)=\varphi_{0} e^{-\xi \theta}, \\
& \alpha(\theta)=\alpha_{0}-\kappa \varphi_{0}\left(e^{-\xi \theta}-1\right),
\end{aligned}
$$

introducing $\kappa=\gamma / \lambda=\zeta / \xi$ as the ratio of the growth $(\zeta=$ $\gamma \tau)$ and damping $(\xi=\lambda \tau)$ factors and denoting initial conditions at $\theta=0$ by $\alpha_{0}$ and $\varphi_{0}$. Eliminating the time dependence leads to

$$
\alpha(\varphi)=\alpha_{0}+\kappa\left(\varphi_{0}-\varphi\right)
$$

which describes the evolution of ice crystal trajectories in phase space. While the fluctuations are damped exponentially, the surface area changes asymptotically by $\Delta \alpha=\kappa \varphi_{0}$ in the direction indicated by the angle, $\beta=\arctan \kappa$. Fast growth rates $\gamma$ and weak damping rates $\lambda$ induce large $\beta$. Figure A1 serves to illustrate the solution behavior for three initial configurations (thick lines with filled symbols). All initial values $\left\{\alpha_{0}, \pm\left|\varphi_{0}\right|\right\}$ (filled symbols) lie on the lines \pm $\left|\varphi_{0}\right|=$ const, each with different values $\alpha_{0}$. They collapse to $\left\{\alpha_{0}+\Delta \alpha, 0\right\}$ (open symbols) by the combined action of depositional growth and damping as indicated by the arrows. The initial width $\Delta \alpha$ is conserved during growth, since the areal growth rate does not depend on $\alpha$.

\section{APPENDIX B}

\section{Analytical Solutions for PDFs}

We examine the probability distribution functions of ice crystal sizes in conditions where crystals either grow or sublimate at a constant rate and of pure supersaturation fluctuations decoupled from ice growth. These PDFs are obtained as analytical solutions of Eq. (4) in the limit of very rapid and very slow growth rates, respectively, and provide some guidance for the interpretation of the results presented in section 3a.

\section{a. Depositional growth at constant supersaturation}

The pure growth equation represents a continuity equation in $\{\theta, \alpha\}$ coordinates with the dimensionless growth rate $d \alpha / d \theta=\gamma \tau s$ :

$$
\frac{\partial P_{\alpha}}{\partial \theta}=\mathcal{L}_{\alpha} P_{\alpha}=-\frac{\partial}{\partial \alpha}\left(\frac{d \alpha}{d \theta} P_{\alpha}\right),
$$

with $P_{\alpha}=P(\alpha, \theta)$. Over time, $P_{\alpha}$ would merely be shifted without a change in shape or width, since $d \alpha / d \theta$ does not depend on $\alpha$. This does not hold for $P_{r}$, as $d r / d t \propto 1 / r$.

Transforming Eq. (B1) into physical $\{r, t\}$ space using $P(r, t) d r=P(\alpha, \theta) d \alpha$ along with $\alpha=\left(r / r_{0}\right)^{2}$ and $\theta=t / \tau$, the growth equation for the PDF of ice particle radii takes the form

$$
\frac{\partial\left(r P_{r}\right)}{\partial t}=-\frac{d r}{d t} \frac{\partial\left(r P_{r}\right)}{\partial r},
$$

where $P_{r}=P(r, t)$ is the ice crystal size PDF determining the probability to find ice particles within the radius range $[r, r+d r]$ at time $t$. The solution $r P_{r}$ is constant along the characteristic curves $r(t)$ that follow from integrating Eq. (1). For an initial $(t=0)$ top-hat distribution that is uniform between the upper and lower boundaries, $r_{01}$ and $r_{02}$, respectively, the solution of Eq. (B2) is given by

$P_{r}(t)=\left\{\begin{array}{ll}\frac{1}{\Delta_{0}} \frac{r}{\sqrt{r^{2}+r_{0}^{2} \gamma s t}:} & r_{1}(t) \leq r \leq r_{2}(t) \\ 0: & \text { else }\end{array}\right.$, 


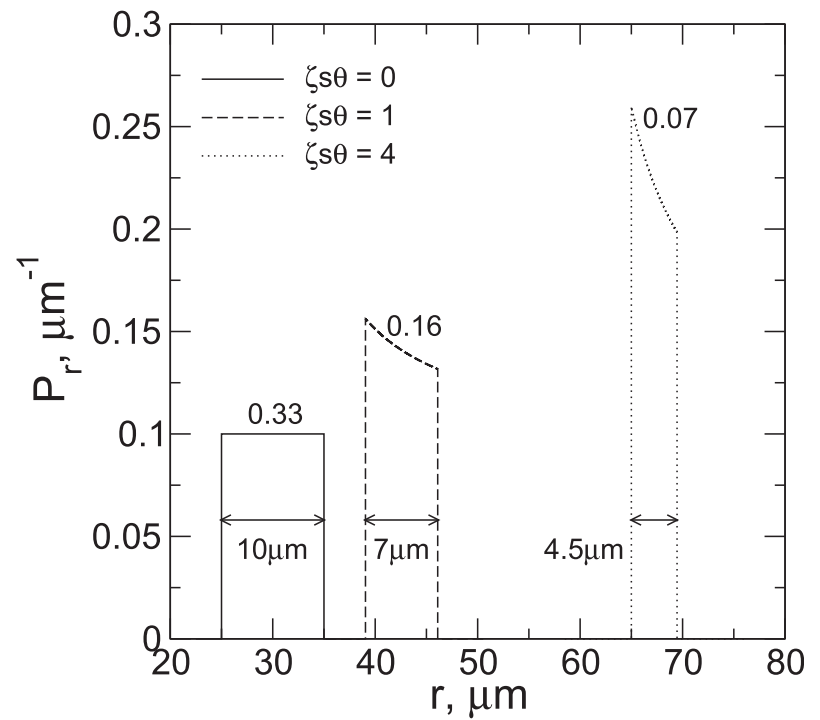

FIG. B1. Temporal evolution of ice particle size PDFs modified by depositional growth at constant supersaturation $(s>0)$. The scaled characteristic growth time is controlled by $\zeta$ s. Solid: initial $\theta=0$, dashed: after $\theta=1 /(\zeta s)$, dotted: after $\theta=4 /(\zeta s)$. The PDFs narrow during growth (but would broaden during sublimation; not shown). The initial top-hat PDF has a width of $10 \mu \mathrm{m}$. Widths are also given for the aged PDFs. Numbers on top of each curve indicate that the spreads (relative size dispersions) of the distributions are defined as the respective ratios of the width and the mean radius.

with the initial distribution width, $\Delta_{0}=r_{02}-r_{01}, r_{0 k}=$ $r_{k}(t=0)$, initial-size PDF $P_{r}(t=0)=1 / \Delta_{0}$, and

$$
r_{k}(t)=r_{0 k} \sqrt{1+\left(r_{0} / r_{0 k}\right)^{2} \gamma s t} \quad k=1,2 .
$$

The width, $\Delta=r_{2}-r_{1}$, changes over time, because the boundaries $r_{k}(t)$ do not grow at the same rate, and $\Delta$ asymptotically approaches zero; hence $P_{r}$ evolves into a monodisperse distribution for $s>0$, and $\int_{r_{1}}^{r_{2}} P_{r} d r=1 \forall t$.

Figure B1 illustrates how an initially uniform PDF (top-hat distribution) of particle sizes narrows and steepens over time if particles grow at a constant supersaturation $(s>0)$, as predicted by Eq. (B3). The probability, as a measure of the ice crystal number, is conserved during depositional growth. These findings hold for any initial-size PDF.

\section{b. Supersaturation fluctuations without ice crystal growth}

The PDF of ice supersaturation driven by damped colored noise follows from

$$
\frac{\partial P_{\varphi}}{\partial \theta}=\mathcal{L}_{\varphi} P_{\varphi}=\xi \frac{\partial\left(\varphi P_{\varphi}\right)}{\partial \varphi}+\mathcal{D} \frac{\partial^{2} P_{\varphi}}{\partial \varphi^{2}}
$$

[appendix C in Benkert et al. (1990)]. The operator $\mathcal{L}_{\varphi}$ consists of a drift term that is proportional to the

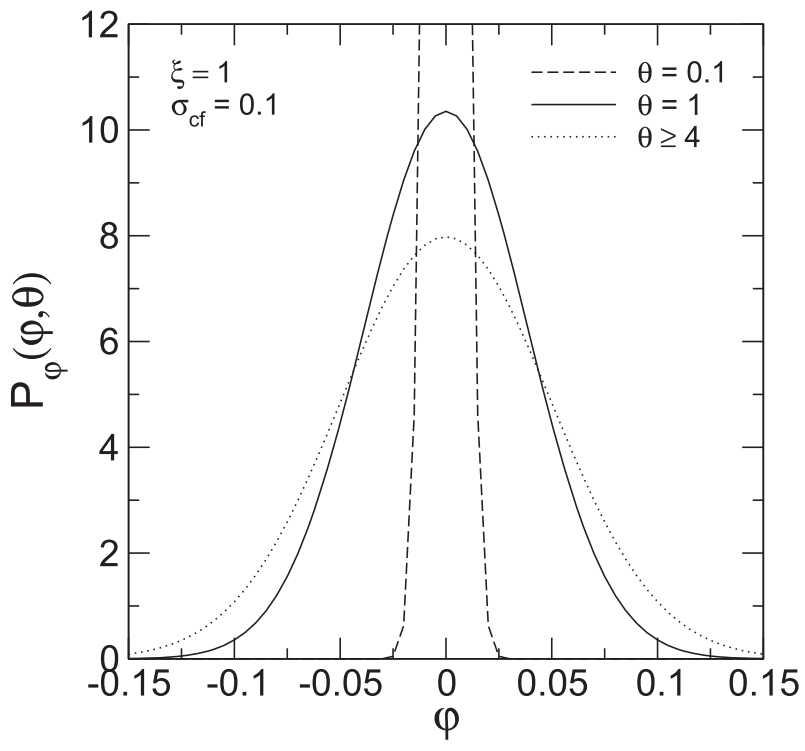

FIG. B2. PDFs of supersaturation fluctuations without depositional growth for various scaled times, $\theta=t / \tau$. The narrow initial distribution evolves into a Gaussian distribution and broadens over time. Asymptotic distributions converge, because cloud ice-induced damping, controlled by $\xi$, causes the distribution variance to approach a stationary value. The asymptotic (damped) fluctuation amplitude (standard deviation) is $\sigma_{\infty}=0.05$.

damping factor $\xi$ arising from the quenching of supersaturation and a diffusion term that is proportional to the time-dependent diffusion coefficient $\mathcal{D}$ arising from the assumption of Gaussian fluctuations.

The solution of Eq. (B5) reads

$$
P_{\varphi}=\frac{1}{\sqrt{2 \pi} \sigma_{\varphi}} \exp \left[-\left(\varphi-\varphi_{0} e^{-\xi \theta}\right)^{2} /\left(2 \sigma_{\varphi}^{2}\right)\right]
$$

with the time-dependent variance

$$
\begin{aligned}
\sigma_{\varphi}^{2}(\theta)= & \frac{\sigma_{\mathrm{cf}}^{2} / 2}{\xi^{2}-1}\left\{1-\frac{1}{\xi}+\left(1+\frac{1}{\xi}\right) \exp (-2 \xi \theta)\right. \\
& -2 \exp [-(\xi+1) \theta]\}
\end{aligned}
$$

This variance increases proportional to $\theta^{2}$ initially and then proportional to $\theta$ asymptotically until it approaches a stationary limit due to ice induced damping:

$$
\sigma_{\varphi}^{2}\left(\theta \gg \theta_{\infty}\right) \equiv \sigma_{\infty}^{2}=\frac{\sigma_{\mathrm{cf}}^{2}}{2 \xi(\xi+1)}
$$

with the dimensionless time scale $(\xi>0)$ 
TABLE C1. List of symbols and acronyms.

\begin{tabular}{|c|c|c|}
\hline Symbol/acronym & Definition & Unit \\
\hline $\mathrm{AV}$ & Anvil cirrus & \\
\hline $\mathrm{CC}$ & Contrail cirrus & \\
\hline ML & Midlatitude cirrus & \\
\hline $\mathrm{TT}$ & Tropical tropopause cirrus & \\
\hline PDF & Probability density function & \\
\hline PSD & Ice crystal number size distribution & \\
\hline 0 & Initial value & \\
\hline$m$ & Mean value (averaged over a PDF) & \\
\hline$\infty$ & Time asymptotic (stationary) value & \\
\hline$\overline{(\cdot)}$ & Large-scale (ensemble) mean value & \\
\hline$e$ & Water vapor saturation pressure over ice & $\mathrm{Pa}$ \\
\hline$k_{B}$ & Boltzmann's constant & $\mathrm{J} \mathrm{K}^{-1}$ \\
\hline$g$ & Acceleration of gravity & $\mathrm{ms}^{-2}$ \\
\hline$n$ & Ice crystal number density & $\mathrm{m}^{-3}$ \\
\hline$p$ & Air pressure & $\mathrm{Pa}$ \\
\hline$r$ & Ice crystal radius (volume-equivalent sphere) & $\mathrm{m}$ \\
\hline$s$ & Ice supersaturation & - \\
\hline$t$ & Time & $\mathrm{s}$ \\
\hline$v_{t}$ & Ice crystal terminal fall speed & $\mathrm{ms} \mathrm{s}^{-1}$ \\
\hline$D$ & Water vapor diffusion coefficient in air & $\mathrm{m}^{2} \mathrm{~s}^{-1}$ \\
\hline$L$ & Maximum dimension of an irregular ice crystal & $\mathrm{m}$ \\
\hline $\mathcal{L}$ & Differential operator in the kinetic Eq. (4) & - \\
\hline$M_{5}$ & Fifth moment of the ice crystal size PDF & - \\
\hline$P$ & (Joint) PDF & - \\
\hline$T$ & Air temperature & $\mathrm{K}$ \\
\hline $\mathcal{D}$ & Supersaturation diffusion coefficient & - \\
\hline$\alpha$ & Scaled ice crystal surface area, $\alpha=\left(r / r_{0}\right)^{2}$ & - \\
\hline$\delta_{r}$ & Size dispersion, $\delta_{r}=\sqrt{\sigma_{r}^{2}} / r_{m}$ & - \\
\hline$\epsilon$ & Stochastic forcing of supersaturation & $\mathrm{s}^{-1}$ \\
\hline$\eta$ & Dynamic viscosity of dry air & $\mathrm{kg} \mathrm{m}^{-1} \mathrm{~s}^{-1}$ \\
\hline$\lambda, \xi$ & Damping parameter, $\xi=\lambda \tau$ & $\mathrm{s}^{-1},-$ \\
\hline$\gamma, \zeta$ & Areal growth parameter, $\zeta=\gamma \tau$ & $\mathrm{s}^{-1},-$ \\
\hline$\kappa$ & Growth-to-damping ratio, $\kappa=\gamma / \lambda$ & - \\
\hline$\nu$ & Volume of an $\mathrm{H}_{2} \mathrm{O}$ molecule in ice & $\mathrm{m}^{3}$ \\
\hline$\rho$ & Bulk mass density of small, compact ice crystals & $\mathrm{kg} \mathrm{m}^{-3}$ \\
\hline$\theta$ & Scaled time, $\theta=t / \tau$ & - \\
\hline$\tau$ & Supersaturation autocorrelation time & s \\
\hline$\varphi$ & Supersaturation fluctuation & - \\
\hline$\sigma_{\mathrm{cf}}$ & Undamped supersaturation fluctuation amplitude & - \\
\hline$\sigma_{r}^{2}$ & Variance of the radial size distribution & $\mathrm{m}^{2}$ \\
\hline$\Delta$ & Increment of a variable in the numerical solver of Eq. (4) & - \\
\hline $\bar{\Phi}$ & Sedimentation ice mass flux & $\mathrm{kg} \mathrm{m}^{-2} \mathrm{~s}^{-1}$ \\
\hline
\end{tabular}

$$
\theta_{\infty}=\max \left[\frac{1}{2 \xi}, \frac{1}{1+\xi}\right]
$$

(Kärcher 2012). For weak damping $(\xi \ll 1)$, this implies $t_{\infty} \simeq 1 /(2 \lambda)$, while for strong damping $(\xi \gg 1)$, the time at which the variance becomes stationary is given by $t_{\infty} \simeq 1 / \lambda$. We note that $\sigma_{\varphi}^{2}$ varies smoothly across $\xi=1$ and that $\sigma_{\infty}^{2}$ exceeds $\sigma_{\text {cf }}^{2}$ for $\xi<0.37$.

Distributions of pure supersaturation fluctuations are displayed in Fig. B2 for various values of scaled times $\theta$. The sharply peaked initial distribution broadens over time and approaches its stationary limit computed using $\sigma_{\text {cf }}=0.1$ and $\xi=1$ (i.e., $\sigma_{\infty}=0.05$ ).

\section{APPENDIX C}

\section{Symbols and Acronyms}

Table C1 shows the symbols and acronyms, along with their definitions and units, used throughout this paper.

\section{REFERENCES}

Bartlett, J. T., and P. R. Jonas, 1972: On the dispersion of the sizes of droplets growing by condensation in turbulent clouds. Quart. J. Roy. Meteor. Soc., 98, 150-164, doi:10.1002/ qj.49709841512. 
Benkert, C., M. O. Scully, A. A. Rangwala, and W. Schleich, 1990: Quantum-noise suppression in lasers via memory-correlation effects. Phys. Rev., 42A, 1503-1514, doi:10.1103/PhysRevA.42.1503.

Brenguier, J.-L., and L. Chaumat, 2001: Droplet spectra broadening in cumulus clouds. Part I. Broadening in adiabatic cores. J. Atmos. Sci., 58, 628-641, doi:10.1175/1520-0469(2001)058<0628: DSBICC $>2.0 . \mathrm{CO} ; 2$.

Burkhardt, U., and B. Kärcher, 2011: Global radiative forcing from contrail cirrus. Nat. Climate Change, 1, 54-58, doi:10.1038/ nclimate1068.

Collins, W. D., and M. Satoh, 2009: Simulating global clouds: Past, present, and future. Clouds in the Perturbed Climate System: Their Relationship to Energy Balance, Atmospheric Dynamics, and Precipitation, J. Heintzenberg and R. J. Charlson, Eds., Strungmann Forum Reports, Vol. 2, MIT Press, 469-486.

Comstock, J. M., T. P. Ackerman, and D. D. Turner, 2004: Evidence of high ice supersaturation in cirrus clouds using ARM Raman lidar measurements. Geophys. Res. Lett., 31, L11106, doi:10.1029/2004GL019705.

Cooper, W. A., 1989: Effects of variable growth histories on droplet size distributions. Part I: Theory. J. Atmos. Sci., 46, 1301-1311, doi:10.1175/1520-0469(1989)046<1301:EOVDGH > 2.0.CO;2.

Davis, S., and Coauthors, 2010: In situ and lidar observations of tropopause subvisible cirrus clouds during TC4. J. Geophys. Res., 115, D00J17, doi:10.1029/2009JD013093.

Dinh, T. P., D. R. Durran, and T. P. Ackerman, 2010: Maintenance of tropical tropopause layer cirrus. J. Geophys. Res., 115, D02104, doi:10.1029/2009JD012735.

Durran, D. R., T. Dinh, M. Ammerman, and T. Ackerman, 2009: The mesoscale dynamics of thin tropical tropopause cirrus. J. Atmos. Sci., 66, 2859-2873, doi:10.1175/2009JAS3046.1.

Feng, Z., X. Dong, B. Xi, S. A. McFarlane, A. Kennedy, B. Lin, and P. Minnis, 2012: Life cycle of midlatitude deep convective systems in a Lagrangian framework. J. Geophys. Res., 117, D23201, doi:10.1029/2012JD018362.

Field, P. R., and A. J. Heymsfield, 2003: Aggregation and scaling of ice crystal size distributions. J. Atmos. Sci., 60, 544-560, doi:10.1175/1520-0469(2003)060<0544:AASOIC >2.0.CO;2.

Fischer, L., C. Kiemle, and G. C. Craig, 2012: Height-resolved variability of midlatitude tropospheric water vapor measured by an airborne lidar. Geophys. Res. Lett., 39, L06803, doi:10.1029/2011GL050621.

Fritts, D. C., L. Wang, and J. A. Werne, 2013: Gravity wave-fine structure interactions. Part I: Influences of fine structure form and orientation on flow evolution and instability. J. Atmos. Sci., 70, 3710-3734, doi:10.1175/JAS-D-13-055.1.

Fueglistaler, S., M. Bonazzola, P. H. Haynes, and T. Peter, 2005: Stratospheric water vapor predicted from the Lagrangian temperature history of air entering the stratosphere in the tropics. J. Geophys. Res., 110, D08107, doi:10.1029/2004JD005516.

Hänggi, P., and P. Jung, 1995: Colored noise in dynamical systems. Advances in Chemical Physics, Vol. 89, I. Prigogine and S. A. Rice, Eds., John Wiley \& Sons, 239-326.

Heymsfield, A. J., 2003: Properties of tropical and midlatitude ice cloud particle ensembles. Part I: Median mass diameters and terminal velocities. J. Atmos. Sci., 60, 2573-2591, doi:10.1175/ 1520-0469(2003)060<2573:POTAMI $>2.0$. CO;2.

Holton, J. R., and A. Gettelman, 2001: Horizontal transport and the dehydration of the stratosphere. Geophys. Res. Lett., 28, 2799-2802, doi:10.1029/2001GL013148.

Hoyle, C. R., B. P. Luo, and T. Peter, 2005: The origin of high ice crystal number densities in cirrus clouds. J. Atmos. Sci., 62, 2568-2579, doi:10.1175/JAS3487.1.
Ivanova, K., and T. P. Ackerman, 2009: Tracking nucleation, growth, and sublimation in cirrus clouds using ARM millimeter-wavelength radar observations. J. Geophys. Res., 114, D06113, doi:10.1029/2008JD010271.

Jeffery, C. A., J. M. Reisner, and M. Andrejczuk, 2007: Another look at stochastic condensation for subgrid cloud modeling: Adiabatic evolution and effects. J. Atmos. Sci., 64, 3949-3968, doi:10.1175/2006JAS2147.1.

Jensen, E. J., O. B. Toon, D. L. Westphal, S. Kinne, and A. J. Heymsfield, 1994: Microphysical modeling of cirrus: 1. Comparison with 1986 FIRE IFO measurements. J. Geophys. Res., 99, 10 421-10 442, doi:10.1029/93JD02334.

, - , H. B. Selkirk, J. D. Spinhirne, and M. R. Schoeberl, 1996: On the formation and persistence of subvisible cirrus clouds near the tropical tropopause. J. Geophys. Res., 101, 21361-21375, doi:10.1029/95JD03575.

- and Coauthors, 2008: Formation of large $(\simeq 100 \mu \mathrm{m})$ ice crystals near the tropical tropopause. Atmos. Chem. Phys., 8, 1621-1633, doi:10.5194/acp-8-1621-2008.

, L. Pfister, and O. B. Toon, 2011: Impact of radiative heating, wind shear, temperature variability, and microphysical processes on the structure and evolution of thin cirrus in the tropical tropopause layer. J. Geophys. Res., 116, D12209, doi:10.1029/2010JD015417.

- and Coauthors, 2013: Ice nucleation and dehydration in the tropical tropopause layer. Proc. Natl. Acad. Sci. USA, 110, 2041-2046, doi:10.1073/pnas.1217104110.

Kahn, B. H., A. Gettelman, E. J. Fetzer, A. Eldering, and C. K. Liang, 2009: Cloudy and clear-sky relative humidity in the upper troposphere observed by the A-train. J. Geophys. Res., 114, D00H02, doi:10.1029/2009JD011738.

Kärcher, B., 2012: Supersaturation fluctuations in cirrus clouds driven by colored noise. J. Atmos. Sci., 69, 435-443, doi:10.1175/JAS-D-11-0151.1.

, and J. Ström, 2003: The roles of dynamical variability and aerosols in cirrus cloud formation. Atmos. Chem. Phys., 3, 823-838, doi:10.5194/acp-3-823-2003.

, and U. Burkhardt, 2008: A cirrus cloud scheme for general circulation models. Quart. J. Roy. Meteor. Soc., 134, 14391461, doi:10.1002/qj.301.

Kay, J. E., M. Baker, and D. Hegg, 2006: Microphysical and dynamical controls on cirrus cloud optical depth distributions. J. Geophys. Res., 111, D24205, doi:10.1029/2005JD006916.

Khvorostyanov, V. I., and K. Sassen, 1998: Cirrus cloud simulation using explicit microphysics and radiation. Part I: Model description. J. Atmos. Sci., 55, 1808-1821, doi:10.1175/ 1520-0469(1998)055<1808:CCSUEM > 2.0.CO;2.

, and J. A. Curry, 1999: Toward the theory of stochastic condensation in clouds. Part I: A general kinetic equation. J. Atmos. Sci., 56, 3985-3996, doi:10.1175/1520-0469(1999)056<3985: TTTOSC $>2.0 . \mathrm{CO} ; 2$.

Korolev, A. V., 1995: The influence of supersaturation fluctuations on droplet size spectra formation. J. Atmos. Sci., 52, 3620-3634, doi:10.1175/1520-0469(1995)052<3620:TIOSFO > 2.0.CO;2.

, and I. P. Mazin, 2003: Supersaturation of water vapor in clouds. J. Atmos. Sci., 60, 2957-2974, doi:10.1175/ 1520-0469(2003)060<2957:SOWVIC >2.0.CO;2.

Kuhs, W. F., C. Sippel, A. Falenty, and T. C. Hansen, 2012: Extent and relevance of stacking disorder in "ice $\mathrm{I}_{\mathrm{c}}$." Proc. Natl. Acad. Sci. USA, 109, 21259-21264, doi:10.1073/ pnas. 1210331110.

Lamquin, N., C. J. Stubenrauch, K. Gierens, U. Burkhardt, and H. Smit, 2012: A global climatology of upper-tropospheric ice 
supersaturation occurrence inferred from the Atmospheric Infrared Sounder calibrated by MOZAIC. Atmos. Chem. Phys., 12, 381-405, doi:10.5194/acp-12-381-2012.

Lawson, R. P., B. Pilson, B. Baker, Q. Mo, E. Jensen, L. Pfister, and P. Bui, 2008: Aircraft measurements of microphysical properties of subvisible cirrus in the tropical tropopause layer. Atmos. Chem. Phys., 8, 1609-1620, doi:10.5194/acp-8-1609-2008.

_ E. Jensen, D. L. Mitchell, B. Baker, Q. Mo, and B. Pilson, 2010: Microphysical and radiative properties of tropical clouds investigated in TC4 and NAMMA. J. Geophys. Res., 115, D00J08, doi:10.1029/2009JD013017.

Levin, L. M., and Y. S. Sedunov, 1966: Stochastic condensation of drops and kinetics of cloud spectrum formation. J. Rech. Atmos., 2, 425-432.

Lin, H., K. J. Noone, J. Ström, and A. J. Heymsfield, 1998: Dynamical influences on cirrus cloud formation process. J. Atmos. Sci., 55, 1940-1949, doi:10.1175/1520-0469(1998)055<1940: DIOCCF $>2.0 . \mathrm{CO} ; 2$.

Liu, Y. S., S. Fueglistaler, and P. H. Haynes, 2010: Advectioncondensation paradigm for stratospheric water vapor. J. Geophys. Res., 115, D24307, doi:10.1029/2010JD014352.

Lohmann, U., and S. E. Schwartz, 2009: Aerosols and clouds in chemical transport models and climate models. Clouds in the Perturbed Climate System: Their Relationship to Energy Balance, Atmospheric Dynamics, and Precipitation, J. Heintzenberg and R. J. Charlson, Eds., Strungmann Forum Reports, Vol. 2, MIT Press, 531-555.

Luo, B. P., and Coauthors, 2003: Ultrathin tropical tropopause clouds (UTTCs): II. Stabilization mechanisms. Atmos. Chem. Phys., 3, 1093-1100, doi:10.5194/acp-3-1093-2003.

Luo, Z., and W. B. Rossow, 2004: Characterizing tropical cirrus life cycle, evolution, and interaction with upper-tropospheric water vapor using Lagrangian trajectory analysis of satellite observations. J. Climate, 17, 4541-4563, doi:10.1175/3222.1.

Mace, G. G., E. E. Clothiaux, and T. P. Ackerman, 2001: The composite characteristic of cirrus clouds: Bulk properties revealed by one year of continuous cloud radar data. J. Climate, 14, 2185-2203, doi:10.1175/1520-0442(2001)014<2185: TCCOCC $>2.0 . \mathrm{CO} ; 2$.

Manton, M. J., 1979: On the broadening of a droplet distribution by turbulence near cloud base. Quart. J. Roy. Meteor. Soc., 105, 899-914, doi:10.1002/qj.49710544613.

Miloshevich, L. M., and A. J. Heymsfield, 1997: A balloon-borne continuous cloud particle replicator for measuring vertical profiles of cloud microphysical properties: Instrument design, performance, and collection efficiency analysis. J. Atmos. Oceanic Technol., 14, 753-768, doi:10.1175/1520-0426(1997)014<0753: $\mathrm{ABBCCP}>2.0 . \mathrm{CO} ; 2$.

Minnis, P., D. F. Young, D. P. Garber, L. Nguyen, W. L. Smith Jr., and R. Palikonda, 1998: Transformation of contrails into cirrus during SUCCESS. Geophys. Res. Lett., 25, 1157-1160, doi:10.1029/97GL03314.

Murphy, D. M., 2003: Dehydration in cold clouds is enhanced by a transition from cubic to hexagonal ice. Geophys. Res. Lett., 30, 2230, doi:10.1029/2003GL018566.

Peter, T., and Coauthors, 2003: Ultrathin tropical tropopause clouds (UTTCs): I. Cloud morphology and occurrence. Atmos. Chem. Phys., 3, 1083-1091, doi:10.5194/acp-3-1083-2003. , C. Marcolli, P. Spichtinger, T. Corti, M. B. Baker, and T. Koop, 2006: When dry air is too humid. Science, 314, 13991402, doi:10.1126/science.1135199.

Pruppacher, H. R., and J. D. Klett, 1997: Microphysics of Clouds and Precipitation. Kluwer Academic, 954 pp.
Prusa, J. M., P. K. Smolarkiewicz, and A. A. Wyszogrodzki, 2008: EULAG, a computational model for multiscale flows. Comput. Fluids, 37, 1193-1207, doi:10.1016/j.compfluid.2007.12.001.

Randel, W. J., and E. J. Jensen, 2013: Physical processes in the tropical tropopause layer and their roles in a changing climate. Nat. Geosci., 6, 169-176, doi:10.1038/ngeo1733.

Risken, H., 1996: The Fokker-Planck Equation: Methods of Solution and Applications. Springer, $472 \mathrm{pp}$.

Sassen, K., D. O'C. Starr, and T. Uttal, 1989: Mesoscale and microscale structure of cirrus clouds: Three case studies. J. Atmos. Sci., 46, 371-396, doi:10.1175/1520-0469(1989)046<0371: MAMSOC $>2.0 . \mathrm{CO} ; 2$.

Smolarkiewicz, P. K., 2006: Multidimensional positive definite advection transport algorithm: An overview. Int. J. Numer. Methods Fluids, 50, 1123-1144, doi:10.1002/fld.1071.

__, and L. G. Margolin, 1998: MPDATA: A finite-difference solver for geophysical flows. J. Comput. Phys., 140, 459-480, doi:10.1006/jcph.1998.5901.

Sölch, I., and B. Kärcher, 2010: A large-eddy model for cirrus clouds with explicit aerosol and ice microphysics and Lagrangian ice particle tracking. Quart. J. Roy. Meteor. Soc., 136, 2074-2093, doi:10.1002/qj.689.

- , and - 2011: Process-oriented large-eddy simulations of a midlatitude cirrus cloud system based on observations. Quart. J. Roy. Meteor. Soc., 137, 374-393, doi:10.1002/qj.764.

Solomon, S., K. H. Rosenlof, R. W. Portmann, J. S. Daniel, S. M. Davis, T. J. Sanford, and G.-K. Plattner, 2010: Contributions of stratospheric water vapor to decadal changes in the rate of global warming. Science, 327, 1219-1223, doi:10.1126/science.1182488.

Stephens, G. L., S.-C. Tsay, P. W. Stackhouse, and P. J. Flatau, 1990: The relevance of microphysical and radiative properties of cirrus clouds to climate and climatic feedback. J. Atmos. Sci., 47, 1742-1753, doi:10.1175/1520-0469(1990)047<1742: TROTMA $>2.0$. CO;2.

Ström, J., B. Strauss, T. Anderson, F. Schröder, J. Heintzenberg, and P. Wendling, 1997: In situ observations of the microphysical properties of young cirrus clouds. J. Atmos. Sci., 54, 2542-2553, doi:10.1175/1520-0469(1997)054<2542:ISOOTM>2.0.CO;2.

Virts, K. S., J. M. Wallace, Q. Fu, and T. P. Ackerman, 2010: Tropical tropopause transition layer cirrus as represented by CALIPSO lidar observations. J. Atmos. Sci., 67, 3113-3129, doi:10.1175/2010JAS3412.1.

Voigt, C., and Coauthors, 2011: Extinction and optical depth of contrails. Geophys. Res. Lett., 38, L11806, doi:10.1029/ 2011 GL047189.

Wang, T., and A. E. Dessler, 2012: Analysis of cirrus in the tropical tropopause layer from CALIPSO and MLS data: A water perspective. J. Geophys. Res., 117, D04211, doi:10.1029/ 2011JD016442.

Winker, D. M., and C. R. Trepte, 1998: Laminar cirrus observed near the tropical tropopause by LITE. Geophys. Res. Lett., 25, 3351-3354, doi:10.1029/98GL01292.

Wood, S. E., M. B. Baker, and D. Calhoun, 2001: New model for the vapor growth of hexagonal ice crystals in the atmosphere. J. Geophys. Res., 106, 4845-4870, doi:10.1029/2000JD900338.

Yang, H., S. Dobbie, G. G. Mace, A. Ross, and M. Quante, 2012: GEWEX Cloud System Study (GCSS) cirrus cloud working group: Development of an observation-based case study for model evaluation. Geosci. Model Dev., 5, 829-843, doi:10.5194/gmd-5-829-2012.

Zhang, C., and J. Y. Harrington, 2014: Including surface kinetic effects in simple models of ice vapor diffusion. J. Atmos. Sci., 71, 372-390, doi:10.1175/JAS-D-13-0103.1. 